\title{
Article
}

\section{Mind Your Outcomes Quality-Centric Systems Development}

\author{
Seyed Hossein HAERI ${ }^{1,2}\left(\mathbb{D}\right.$, Peter Thompson $\left.{ }^{3}{ }^{(}\right)$, Neil Davies ${ }^{3}\left(\mathbb{D}\right.$, Peter Van Roy ${ }^{4} \mathbb{D}$, Kevin \\ Hammond $^{5}$ (D) James Chapman ${ }^{5}$ (i) \\ Formal Methods Team, IOHK; hossein.haeri@iohk.io \\ Department of Informatics, University of Bergen, Norway; hossein@uib.no \\ Predictable Network Solutions Limited, Stonehouse, U.K.; [Peter.Thompson,Neil.Davies]@pnsol.com \\ Université catholique de Louvain, Belgium; pvr@info.ucl.ac.be \\ 5 IO Global; [kevin.hammond,james.chapman]@iohk.io \\ * Correspondence: hossein.haeri@iohk.io \\ + This paper is an extended version of our paper published in $33^{\text {rd }}$ Symposium on Implementation and \\ Application of Functional Languages (IFL21), as a draft.
}

\begin{abstract}
This paper directly addresses a critical issue that affects the development of many complex distributed software systems: how to establish quickly, cheaply and reliably whether they will deliver their intended performance before expending significant time, effort and money on detailed design and implementation. We describe $\triangle \mathrm{QSD}$, a novel metrics-based and quality-centric paradigm that uses formalised outcome diagrams to explore the performance consequences of design decisions, as a performance blueprint of the system. The $\triangle$ QSD paradigm is both effective and generic: it allows values from various sources to be combined in a rigorous way, so that approximate results can be obtained quickly and subsequently refined. $\triangle$ QSD has been successfully used by Predictable Network Solutions for consultancy on large-scale applications in a number of industries, including telecommunications, avionics, and space and defence, resulting in cumulative savings of $\$ B$. The paper outlines the $\triangle$ QSD paradigm, describes its formal underpinnings, and illustrates its use via a topical real-world example taken from the blockchain/cryptocurrency domain, where application of this approach enabled an advanced distributed proof-of-stake system to meet challenging throughput targets.
\end{abstract}

Keywords: formal semantics; quality attenuation; distributed systems; system design; scalability; performance; feasibility; blockchain; $\Delta \mathrm{Q}$.

\section{Introduction}

The modern world depends on large-scale software systems to run its critical infrastructure: telecommunications, energy, finance, transport, government, the military, and major multi-national companies all rely on correct and reliable software to carry out their day-to-day operations. Individuals in many countries increasingly rely on online systems for many aspects of their daily lives. Such systems are complex and expensive to construct and maintain. All too often, however, they fail to deliver the intended performance, or sometimes even to meet the most basic requirements of reliability and usability. One example of the economic impact of failing to adequately manage performance is OnLive. OnLive was a cloud gaming platform that attempted to deliver high quality video and real-time interactivity, but failed to deliver an acceptable experience [1]. As a consequence, the company folded, wiping out a $\$ 1.8 \mathrm{~B}$ valuation and costing investors several hundred million US dollars $[2,3]$.

Such results are, fundamentally, a failure both of design and of management. This has a major social and economic cost: fixing a problem in development typically costs 10 times as much as fixing it in design, and fixing a problem in a released product typically costs 100 times as much as fixing it in development [4]. Overall, the cost of failed software projects in the US in 2020 was approximately \$260B (up from \$177.5B in 2018) [5]. 
One core cause of problems such as these is that modern software development practices successfully emphasise rapid and flexible software construction, but fail to adequately consider essential quality requirements, or even to consider properly whether a system can actually meet its intended outcomes, particularly when deployed at scale. In complex system development there is a tendency for cost/performance hazards to appear late in the System Development Life Cycle (SDLC). Unfortunately, such issues can invalidate design choices, requiring major redesign or implementation changes. This can lead to time and cost overruns, and sometimes project cancellation. Thus there is an urgent need for verification and validation of resource cost and system performance (as opposed to simply functional correctness), and for this to be part of an ongoing design process rather than applied late in the development. This process must be applicable to distributed and complex hierarchical systems, and support both initial and incremental development. This methodology becomes increasingly important as such systems are used for cyber-physical, mission-critical or even safety-of-life applications. Several factors make this task more difficult:

1. System requirements are often vague and/or contradictory, and can change both during and after development;

2. Complexity forces hierarchical decomposition of the problem, creating boundaries, including commercial boundaries with third-party suppliers, that may hinder optimal development and obscure risks;

3. Time pressure forces parallel development that may be at odds with that hierarchical decomposition, and encourages leaving 'tricky' issues for later, when they tend to cause re-work and overruns, and leave tail-risks;

4. Cost and resource constraints force resources to be shared, both within the system and with other systems (e.g., when network infrastructure or computing resources are shared); they may also require re-use of existing assets (own or third-party), introducing a degree of variability in the delivered performance;

5. The performance of particular components or subsystems may be incompletely quantified;

6. System performance and resource consumption may not scale linearly (which may not become apparent until moving from a lab/pilot phase to a wider deployment);

7. At scale, exceptional events (transient communications and/or hardware issues) can no longer be treated as negligibly rare and their effects and mitigation need to be considered, along with the associated performance impacts.

Thus, what is needed is: (1) a way of capturing performance and resource requirements that accommodates all the various sources of uncertainty; and, (2) a process for decomposing a top-level requirement into subsystem requirements that provides confidence that satisfying all the lower-level requirements will also satisfy the top-level one. For functional aspects of system behaviour there are various ways of dealing with this [6]. In terms of performance - which for time-critical systems design means delivering an outcome within a time bound (not just at a particular rate) - there are established approaches [7], but these all have significant limitations.

This paper directly addresses those issues by defining the $\triangle$ QSD systems development paradigm and providing a high-level formalism that can be used throughout the software development process. It illustrates the use of $\triangle Q S D$ on an actual real-time distributed system, namely the Cardano blockchain diffusion algorithm. $\triangle \mathrm{QSD}$ is a quality-centric paradigm, focusing on meeting timeliness constraints and acceptable failure rate of the top-level outcomes with acceptable resource consumption. The paradigm has been used successfully by Predictable Network Solutions in many large industrial projects, collectively saving billions of dollars and person-centuries of development effort. It informs high-level management and system design decisions by showing where conflicts exist (or may exist) between system designs and required outcomes. $\triangle \mathrm{QSD}$ presents the design process as a sequence of refinement steps, that can be taken all the way from very high level to a fully specified and constructible system. It is able to compute predicted performance at any stage of the design process, where performance is seen broadly as comprising timeliness, behaviour under load, resource consumption, 
and other key system metrics. Central to $\triangle \mathrm{QSD}$ is the concept of an outcome, defined as a specific system behaviour with specified start and end points, and localised in space and time. In $\triangle Q S D$, the system engineer models the system as an outcome diagram, which is a graph that captures the causal relationships between system outcomes.

\subsection{Main Contributions of this Paper}

The main contributions of this paper are to:

1. Introduce $\triangle \mathrm{QSD}$, a formalism (Section 5) focused on rapidly exploring the performance consequences of design and implementation choices, where:

(a) performance is a first class citizen, ensuring that we can focus on details relevant to performance behaviour;

(b) the whole software development process is supported, from checking feasibility of initial requirements to making decisions about subtle implementation choices and potential optimisations;

(c) we can measure our choices against desired outcomes for individual users (customer experience);

(d) analysis of saturated systems is supported;

(e) analysis of failure is supported.

We use term-rewriting for formalising refinements (Definition 3 in Section 5) and denotational semantics for formalising timeliness analysis (Section 5.3) as well as load analysis (Section 5.4).

2. Describe key decisions made in the development process of a real system - i.e., the Cardano blockchain, presented as a running example - and show how $\triangle$ QSD is able to quickly rule out infeasible decisions, predict behaviour, and indicate design headroom (slack) to decision makers, architects, and developers (Section 4).

While the $\Delta Q$ concept has been described in earlier papers [8] [9], and used to inform a number of large-scale system designs, these previous contributions have only used it in an informal manner. By providing a formal definition of $\triangle \mathrm{QSD}$, and showing how it can be used in practice, we are taking an important step towards a general evidence-based engineering methodology for developing real-time distributed systems.

As described above, a key contribution of this work is the new formalism of $\triangle Q S D$ that defines a system design as a sequence of outcome diagrams. This sequence starts with a fully unspecified system and ends either with a fully specified system or a convincingly specified-enough system (both deemed as constructible), or with the conclusion that the system goals are infeasible. The formalism allows exploration of the design space by assessing the consequences of the decisions that are taken at each refinement step and possibly retracting, giving rise to threaded decision trees. For each partially specified system, we compute the predicted timeliness and behaviour and resource consumption of the system under load, obtaining one of three possible conclusions: (1) infeasibility - hence, ceasing further development and revising former design decisions; (2) slack - hence, ceasing further optimisation because the system is good enough; or (3) indecisiveness - hence, requiring additional scrutiny until one of the alternative conclusions can be drawn. The paper gives one large example, blockchain diffusion, that illustrates how $\triangle$ QSD can be used in practice, explaining how the formalism can be used to drive the design process and associated decision making. This example is a real-world application that is in continuous use as a core part of the Cardano blockchain technology (https://cardano.org/).

The requirement that most approaches impose for fully-specified, or even fully-implemented, systems is a serious disadvantage, since it does not allow the properties of subsystems to be encapsulated and hierarchically (de)composed. Compositionality, the principle that the meaning of a complex expression is determined by the meanings of its constituent expressions and the rules that are used to combine them, is key to managing complexity within the systems development life-cycle. For compositional properties, what is "true" about subsystems (e.g., their timeliness, their resource 
consumption) is also "true" about their (appropriate) combination: there exists an invariant (e.g., timeliness, aspects of functional correctness) that must hold over the reified components of the system. In the broader software development space, functional programming techniques are improving the compositionality of functional aspects of software systems, and can deliver high assurance of functional correctness when combined with appropriate formal methods [10]. The paradigm presented here, which we call $\triangle Q S D$, represents a similar step change in handling the "non-functional" aspects of performance and resource consumption. By treating delay and failure as a single object, called 'quality attenuation', our paradigm can be thought of as a combination of passage time analysis and failure mode effects analysis (FMEA).

\subsection{Structure of the Paper}

This paper has the following structure:

1. Section 3 defines the basic concepts that underlie the $\triangle$ QSD formalism: outcomes, outcome diagrams, and quality attenuation $(\Delta Q)$. We also compare outcome diagrams with more traditional diagrams such as block diagrams.

2. Section 4 gives a realistic example of the $\triangle$ QSD paradigm, showing a step-by-step design of block diffusion based on quality analysis. (Block diffusion itself is introduced in Section 2.) This example introduces the basic operations of $\triangle Q S D$ in a tutorial fashion. The example uses realistic system parameters that allow us to compute predicted system behaviour.

3. Section 5 gives the formal mathematical definition of $\triangle$ QSD and its main properties. With this formal definition, it is possible to validate the computations that are used by $\triangle \mathrm{QSD}$ as well as to build tools based on $\triangle$ QSD.

4. Section 6 gives a comprehensive discussion about related work from three different viewpoints: theoretical approaches for performance analysis (Section 6.1), performance design practices in distributed systems (Section 6.2), and programming languages and software engineering (Section 6.3).

5. Section 7 summarises our conclusions, and describes our plans to further validate $\triangle Q S D$ and to build a dedicated toolset for real-time distributed systems design that builds on the $\triangle \mathrm{QSD}$ paradigm. 


\section{Running Example: Block Diffusion in the Cardano Blockchain}

A blockchain is a form of distributed ledger. It comprises a number of blocks of data, each of which provides a cryptographic witness to the correctness of the preceding blocks, back to some original 'genesis' block (a 'chain' of blocks, hence 'blockchain') [11]. Nodes in the system use some specified protocol to arrive at a distributed consensus as to the correct sequence of blocks, even in the presence of one or more 'adversaries' that aim to convince other nodes that a different sequence is correct. One such consensus protocol is Ouroboros Praos [12], which underpins Cardano (https: / www.cardano.org), one of the world's leading cryptocurrencies. Ouroboros Praos uses the distribution of 'stake' in the system (i.e. the value of the cryptocurrency tokens that are controlled by each node) to randomly determine which node (if any) is authorised to produce a new block in the chain during a specific time interval ( $\mathrm{a}$ 'slot'); the more stake a node controls, the more likely it is to be authorised to produce a block. For this to be effective, it is important that the block-producing node has a copy of the most recently produced block, so that the new block can correctly extend the existing chain. Since the block producer is selected at random, this means that the previous block needs to have been copied to all block-producing nodes; we call this process 'block diffusion'. Since blocks are produced on a predetermined schedule and each block depends on its predecessor, block diffusion is a hard real-time problem; each block must be diffused before the next block can be produced. In order to be robust, however, the consensus algorithm is designed to withstand some imperfections in block diffusion, hence the effective requirement is that blocks should be well-diffused "sufficiently often". Put another way, the probability that a block fails to arrive in time for the production of the next block must be suitably bounded. The engineering challenge is to quantify this probability as a function of the design and of the parameter choices of the implementation.

The scale of the challenge is illustrated by Cardano. Cardano is a global-scale distributed system that eschews centralised management. At the time of writing, 2,948 globally-distributed nodes cooperate to produce and distribute blocks for $\$ 45.77 \mathrm{~B}$ of cryptocurrency that is associated with 956,092 distinct user addresses. The stake distribution at the time of writing is shown in Fig. 1.

In Cardano, slots are one second long and blocks are produced every 20 seconds on average. An initial implementation of Cardano (code-named 'Byron') was functionally correct but proved incapable

肃

Live blocks vs Stake

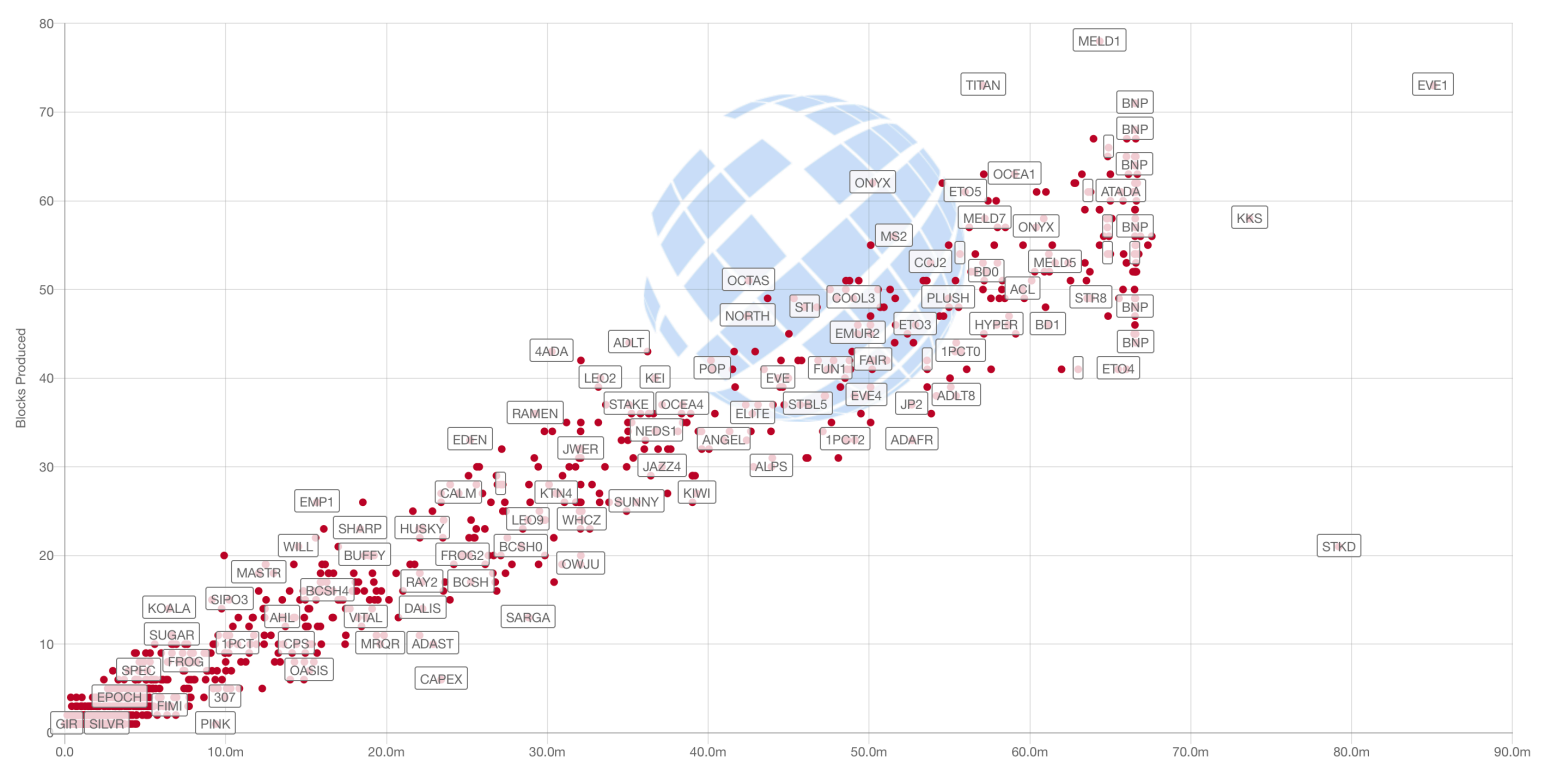

Figure 1. Distribution of Stake by Node on the Cardano Blockchain, November 2021 (source: https: / / pooltool.io/analysis). The y-axis represents the number of blocks produced by each "stake pool"; the $x$-axis represents the stake that is held by the pool (in Ada). 
of effective block diffusion without rigid control of the nodes and their topology; a re-implementation (called 'Jormungandr') targeted higher performance by using a different programming language (Rust instead of Haskell), but this also missed the block diffusion target by a wide margin. A further, and ultimately successful, re-implementation (called 'Shelley' [13]), used Haskell to retain strong correctness assurances, but applied the principles that are discussed in this paper to also ensure adequate performance in a fully decentralised deployment.

\subsection{Key Design Decisions}

In the design of Shelley, a number of inter-related decisions had to be made. These included:

How are nodes connected? It might seem that connecting every node to every other would minimise block diffusion time, however the lack of any control over the number and capabilities of nodes makes this infeasible. Nodes can only be connected to a limited number of peer nodes; the number of connected peers and how they are chosen then become important.

How much data should be in a block? Increasing the amount of data in a block improves the overall throughput of the system but makes block diffusion slower.

How should blocks be forwarded? Simply forwarding a new block to all connected nodes would seem to minimise delay, but this wastes resources, since a node may receive the same block from multiple peers. In the extreme case, this represents a potential denial-of-service attack. Splitting a block into a small header portion (sufficient for a node to decide whether it is new) and a larger body that a node can choose to download if it wishes mitigates this problem but adds an additional step into the forwarding process.

How much time can be spent processing a block? Validating the contents of a block before forwarding it mitigates adversarial behaviour, but can be computationally intensive, since the contents may be programs that need to be executed (called 'smart contracts'); allowing more time for such processing permits more, and more complex, programs but makes block diffusion slower.

The remainder of this paper shows how such design decisions can be quantified using the $\Delta \mathrm{QSD}$ paradigm.

\subsection{Formulating the Problem}

We assume that a collection of blockchain nodes is assembled into a random graph (randomness is important in a blockchain setting for mitigating certain adversarial behaviours). In each time slot, a randomly-chosen node may generate a block, and we are interested in the probability that the next randomly-chosen node has received that block before it generates the next block.

\section{Problem Statement}

Starting from blockchain node $A$, what is the probability distribution of the time taken for a block to reach a different node $Z$, when $A$ and $Z$ are picked at random from the graph?

Since the graph is random with some limited node degree $N$, there is a strong chance that $A$ is not directly connected to $Z$, and so the block will have to pass through a sequence of intermediate nodes $B, C, \ldots$ The length of this sequence is a function of the size and node degree of the graph [14]. The (distribution of) time to forward a block directly from one node to another is known (e.g., by measurement). 


\section{Foundations}

In the remainder of this paper, we will take the system of discourse to be fixed for the design engineer. We assume that this system has a number of tasks that must be performed. In order to perform a task that is not considered to be atomic by the design engineer, the system might need to perform several other sub-tasks. The process of clarifying the details of the system by breaking higher-level tasks into such sub-tasks is what we call refinement (Definition 3 in Section 5). By refining a system, one goes from a coarser granularity of the design to a finer one. (See Sections 4.1-4.3 for examples of that.) Sometimes, the design engineer will return to a coarser grained design (the dashed grey arrows in Fig. 6) in order to take a different direction of refinement. (See Sections 4.4-4.7 for example.) Some reasons why the might want to do that are: to investigate other aspects of their system; to compare two alternative design choices; or because a refinement fails to meet the necessary performance or other requirements. $\triangle \mathrm{QSD}$ is thus design exploration in the world of refinements.

This section sets the stage for presenting design exploration in action (Section 4) by introducing the fundamental concepts: Outcomes (Section 3.1), Outcome Diagrams (Section 3.2), and Quality Attenuation (Section 3.3). It then, gives a simple example of how to approach problems à la $\triangle Q S D$ (Section 3.4). This section ends in a discussion on why $\triangle$ QSD advises a new diagram in the presence of all the existing ones in Software Engineering (Section 3.5).

\subsection{Outcomes}

An outcome is what the system obtains by performing one of its tasks. Each task has precisely one corresponding outcome and each outcome has precisely one corresponding task. We say that an outcome is 'performed' to mean that the corresponding task of an outcome is performed. Likewise, we might use task adjectives for outcomes too, even though outcomes and tasks are inherently different. For example, by an atomic outcome, we mean an outcome whose corresponding task is itself atomic.

We take an event-based perspective, in which each outcome has two distinct sets of events: the starting set of events (any one of which must happen before the task can commence) and the terminating set of events (at least one of which must happen before the task can be considered complete). Each of those sets consists of events that are of particular interest (as opposed to just any event). We call such events of interest the observables. For example, an observable in the starting set, $S_{o}$, of an outcome $o$ is of interest because it signifies the point in time at which $o^{\prime}$ s performance began as well as the 3D location of $o$. Collectively, these comprise the $4 \mathrm{D}$ location or simply location of $o$. Likewise, an observable from the terminating set, $T_{o}$ of $o$ is an event that contains information regarding the location of $o$. While it may seem unusual to refer explicitly to location in a computer science context, when considering distributed systems, the outcomes of interest are precisely those that begin at one location and finish at another. Of course, once an observable from $S_{o}$ occurs, there is no guarantee that one from $T_{o}$ will occur within $o^{\prime}$ s duration limit, $d(o)$ (i.e., the relative time by which $o$ is required to complete). However, when an observable $T_{0}$ does occur within the duration limit after one from $S_{o}, o$ is said to be done.

Diagrammatically, we show an outcome using an orange circle. As shown in Figure 2 we depict the starting set and the terminating set of an outcome using small boxes to the left and right of the outcome's circle, respectively. The starting set is connected to the outcome from the left and the terminating set from the right. When they are unimportant for an outcome, we do not include the starting set and the terminating set of that particular outcome in the diagram.

We consider one special kind of outcome. Consider the situation where a design engineer is aware that an outcome is not atomic. They will eventually need to further break the outcome into its sub-outcomes. Nevertheless, the current level of granularity is sufficient to carry out a particular analysis (see Section 5.3 and Section 5.4 for two example analyses). In $\triangle$ QSD, a black box can be used for that particular outcome. Black boxes are those outcomes that either:

1. Can be easily quantified without even a need for them to be named; 


\section{$\square-\square$}

Figure 2. An Outcome with its Starting Set and Terminating Set on its Left and Right

2. Are beyond the design engineer's control (and so may need to be quantified by external specification or measurement); or,

3. Are ones for which the design engineer has intentionally left the details for later.

Outcome variables are the variables that we use to refer to a given outcome.

\subsection{Outcome Diagrams and Outcome Expressions}

The description of a system in terms of its outcomes requires the causal relationships between the outcomes to be captured. In $\triangle \mathrm{QSD}$, these relationships are captured in outcome diagrams. In addition to its graphical presentation, each outcome diagram can be presented algebraically, using its corresponding outcome expression. As shown in Figure 3, outcome diagrams offer four different ways to describe the relationships between outcomes.

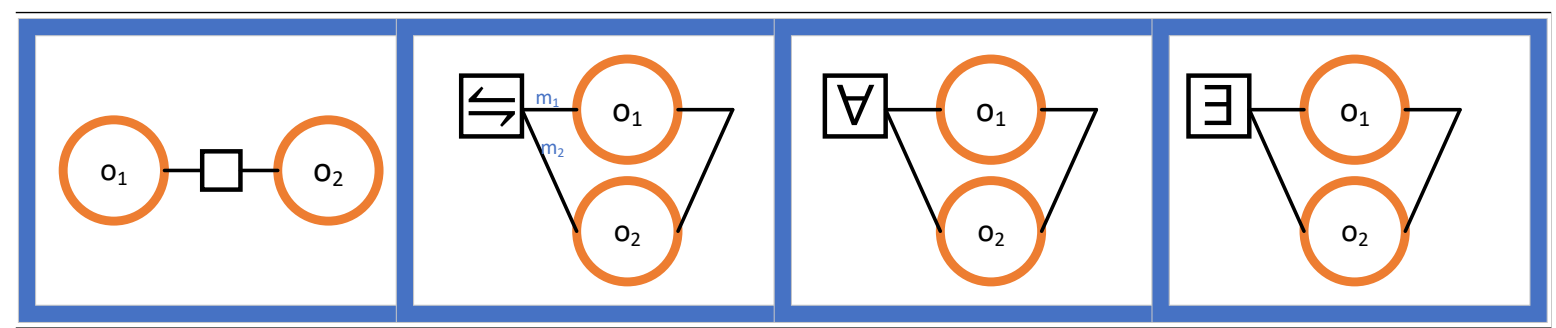

Figure 3. Relationships in an Outcome Diagram. From Left to Right: 1) Sequential Composition; 2) Probabilistic Choice; 3) All-to-Finish; 4) First-to-Finish.

We now explain Figure 3 from left to right.

- In the first case, the outcomes $o_{1}$ and $o_{2}$ are said to be sequentially composed. $o_{2}$ therefore causally depends on $o_{1}$. We maintain a directional convention to avoid showing directions explicitly: when an edge connects two outcomes, the right one causally depends on the left one. The corresponding outcome expression is " $\mathrm{O}_{1} \bullet>\bullet \mathrm{O}_{2}$."

- In the second case, a probabilistic choice is made between $o_{1}$ and $o_{2}$. Notice the weights $m_{1}$ and $m_{2}$. The outcome of the choice is the same as $o_{1}$ with probability $\frac{m_{1}}{m_{1}+m_{2}}$ and the same as $o_{2}$ with probability $\frac{m_{2}}{m_{1}+m_{2}}$. The corresponding outcome expression is " $o_{1} \frac{m_{1}}{m_{2}} o_{2} . "$

- In the third case, an all-to-finish (aka last-to-finish) combination is produced from $o_{1}$ and $o_{2}$. For two outcomes $o_{1}$ and $o_{2}$ that are started at the same time and that are run in parallel, the outcome is done when both $o_{1}$ and $o_{2}$ are done. The corresponding outcome expression is " $\forall\left(o_{1} \| o_{2}\right)$."

- In the final case, a first-to-finish combination is produced from $o_{1}$ and $o_{2}$. For two outcomes $o_{1}$ and $o_{2}$ that are started at the same time and that are run in parallel, the outcome is done when either $o_{1}$ or $o_{2}$ is done. The corresponding outcome expression is " $\exists\left(o_{1} \| o_{2}\right)$." 


\subsection{Quality Attenuation $(\Delta Q)$}

From the perspective of a user, a perfect system would deliver the desired outcome without error, failure or delay, whereas real systems always fall short of this; we can say that the quality of their response is attenuated relative to the ideal. We denote this 'quality attenuation' by the symbol $\Delta Q$ and reformulate the problem of managing performance as one of maintaining suitable bounds on $\Delta \mathrm{Q}$ [15]. This is an important conceptual shift because 'performance' may appear to be something that can be increased arbitrarily, whereas $\Delta \mathrm{Q}$ (rather like noise) is something that may be minimised but that can never be completely eliminated. Indeed, some aspects of $\Delta Q$, such as the time for signals to propagate between components of a distributed system, cannot be reduced below a certain point.

Because the response of the system in any particular instance can depend on a wide range of factors, including the availability of shared resources, we model $\Delta \mathrm{Q}$ as a random variable. This allows various sources of uncertainty to be captured and modelled, ranging from as-yet-undecided aspects of the design, to resource use by other processes, to behavioural dependence on data values.

In capturing the deviation from ideal behaviour, $\Delta Q$ incorporates both delay (a continuous random variable) and exceptions/failures (discrete variables). This can be modelled mathematically using Improper Random Variables (IRVs), whose total probability is less than one [16]. If we write $\Delta \mathrm{Q}(\mathrm{x})$ for the probability that an outcome occurs in a time $t \leq x$, then we can define the 'intangible mass' of such an IRV as $1-\lim _{x \rightarrow \infty} \Delta Q(x)$. In $\Delta Q$, this encodes the probability of exception or failure. This is illustrated in Figure 4, which shows the cumulative distribution function (CDF) of an IRV (with arbitrary time units).

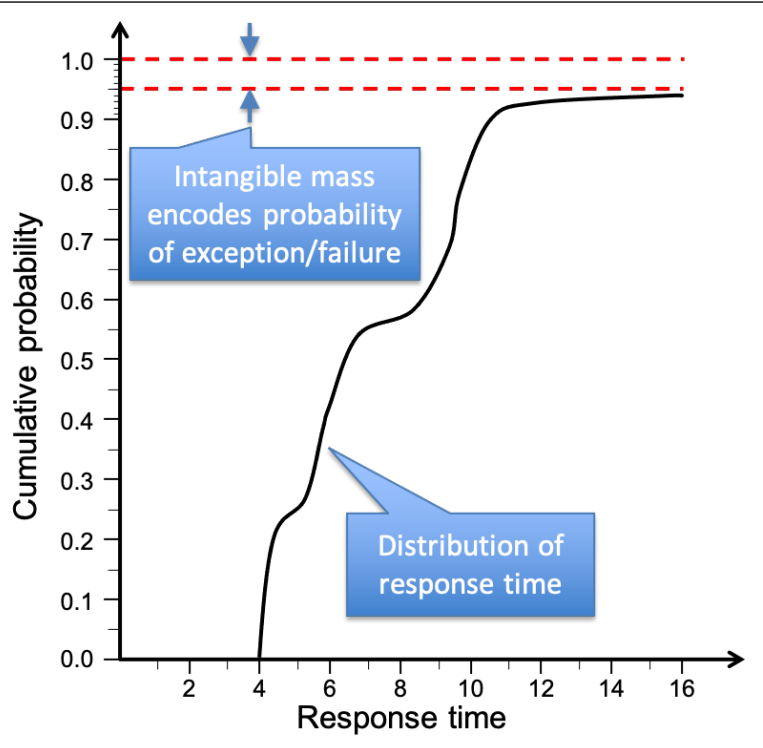

Figure 4. Cumulative Distribution of an Improper Random Variable (IRV).

We can define a partial order on such variables, in which the 'smaller' attenuation is the one that delivers a higher probability of completing the outcome in any given time:

$$
\left(\forall_{x} \Delta Q_{1}(x) \geq \Delta Q_{2}(x)\right) \equiv \Delta Q_{1} \leq \Delta Q_{2}
$$

This partial order has a 'top' element, which is simply perfect performance: $\top \equiv\left(\forall_{x} \Delta Q(x)=1\right)$, and a 'bottom' element, which is total failure (an outcome that never occurs): $\perp \equiv\left(\forall_{x} \Delta Q(x)=0\right)$. We can write specifications for system performance using this partial order by requiring the overall $\Delta \mathrm{Q}$ to be less than or equal to a predefined bounding case. Where the $\Delta \mathrm{Q}$ is strictly less than the requirement, we say there is performance slack; when it is strictly greater than the requirement, we say there is a performance hazard. (Cf. Definitions 5 and 8.) 
Assessments might also find the current level of information about a system to be indecisive neither slack nor hazard. The simplest reason for indecisiveness is the partiality of $\leq$ in Equation (1). Another reason for indecisiveness might be conflict between different analyses. For example, timeliness analysis (Section 5.3) might show slack whilst load analysis (Section 5.4) shows hazard. A third reason might be that, even though the formulations end up indicating slack or hazard, the system is still detailed so little the result of the analysis should not be counted on.

The relationships between outcomes that were shown in Figure 3 then induce corresponding relationships between the $\Delta \mathrm{Qs}$ of those outcomes, as explained in Section 5.3. The key to the compositionality of the approach is that the partial order is preserved by the operations that combine $\Delta$ Qs. Thus, for example

$$
\Delta \mathrm{Q}_{1} \leq \Delta \mathrm{Q}_{2} \Longrightarrow \Delta \mathrm{Q}_{1} \oplus \Delta \mathrm{Q}_{3} \leq \Delta \mathrm{Q}_{2} \oplus \Delta \mathrm{Q}_{3}
$$

This enables an overall timeliness requirement to be broken into 'budgets' for sub-outcomes. More details of this approach are given in [17].

\subsection{Simple Example}

Consider the simple distributed system of a web browser interacting with a set of servers that collectively provide a web page. The outcome that is of interest to the user starts with the event of clicking on a URL, and ends with the event of the page being fully rendered. This corresponds to the first row of Figure 5. The second row shows the distinction between the user and the browser, and the third row exposes the back-end servers. A typical web page will contain a variety of elements that are served by servers from different host domains. So, for each element, the browser (and its supporting $\mathrm{O} / \mathrm{S}$ ) must first resolve the corresponding domain name, then establish a connection to the given server, and finally download and then render the provided content. Thus for each element that needs to be displayed, the $\Delta \mathrm{Q}$ is the sequential composition of the $\Delta \mathrm{Qs}$ of the component steps described above; and the $\Delta \mathrm{Q}$ of rendering the whole page is an all-to-finish combination of the $\Delta \mathrm{Qs}$ of all the elements. Note that this formulation automatically deals with the possibility that any of the steps may fail, and provides the resultant failure probability for the whole process in addition to the distribution of expected completion times.

This simple model can be further refined, as needed to meet real-world requirements. For example, DNS resolution might provide alternative server addresses for load-balancing purposes, and each of these servers might have different $\Delta$ Qs when providing the same content to the user (perhaps because they are located in different geographical locations, or are provisioned using systems with different CPU or storage capabilities). We can represent this as a probabilistic choice between these outcomes, weighted by the probability that a specific server is used. This weights the corresponding $\Delta Q$. In addition, we might also consider the effect of load and contention for shared resources, for example network interface bandwidth or rendering capacity, or the impact of different DNS caching architectures on performance. These aspects of system performance design are formalised in Section 5 . 


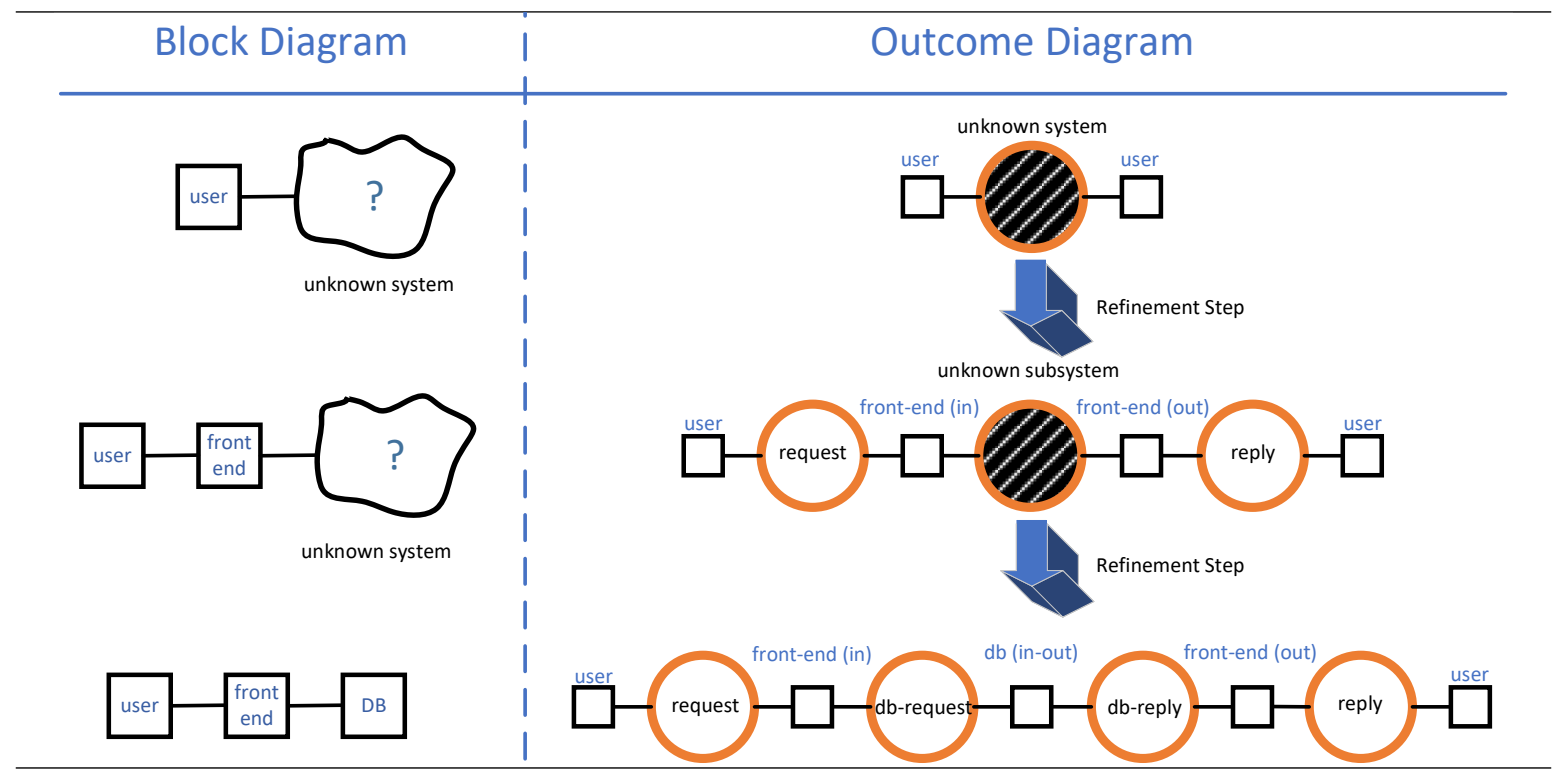

Figure 5. Block Diagram and Outcome Diagram for a Simple System Constructed using Stepwise Refinement.

\subsection{Alternatives to Outcome Diagrams — Why a New Diagram?}

The $\triangle$ QSD paradigm introduces the concept of outcome diagrams. It is perfectly reasonable to ask at this point: "Why another diagram? What is it that outcome diagrams capture that UML diagrams, for example, cannot?" Let us answer these questions by comparing outcome diagrams with UML. We first recall the two main properties of outcome diagrams in the $\triangle$ QSD paradigm:

- An outcome diagram specifies the causal relations between outcomes. An outcome is a specific system behaviour defined by its possible starting events and its possible terminating events. For example, sending a message to a server is an outcome defined by the beginning of the send operation and the end of the send operation. The action of sending a message and receiving a reply is observed as an outcome, defined by the beginning of the send operation and the end of receive operation. Outcomes can be decomposed into smaller outcomes, and outcomes can be causally related. For example, the send-receive outcome can be seen as a causal sequence of a send outcome and a receive outcome.

- An outcome diagram can be defined for a partially specified system. Such an outcome diagram can contain undefined outcomes, which are called black boxes. A black box does not correspond to any defined part of the system, but it still has timeliness and resource constraints. Refining an outcome diagram can consist in replacing one of its black boxes with a subgraph of outcomes.

A crucial property of an outcome diagram is that it is an observational concept. That is, it says something about what a user observes of a system from the outside, but it does not say anything about how the system is constructed internally. 


\subsubsection{UML Diagrams}

UML is a rich language defined to model many different aspects of software, including its structure, behavior, and the processes it is part of. The UML 2 standard defines 14 kinds of diagrams, which are classified into structural diagrams and behavioural diagrams. We first note two general properties of outcome diagrams that UML diagrams do not share:

- Observational property All UML diagrams, structural and behavioural, define what happens inside the system being modeled, whereas outcome diagrams define observations from outside the system. The outcome diagram makes no assumptions about the system's components or internal states.

- Wide coverage property It is possible for both UML diagrams and outcome diagrams to give partial information about a system, so that they correspond to many possible systems. As long as the systems are consistent with the information in the diagram, they will have the same diagram. However, an outcome diagram corresponds to a much larger set of possible systems than a UML diagram. For an outcome diagram, a system corresponds if it has the same outcomes, independent of its internal structure or behaviour. For a UML diagram, a system corresponds if its internal structure or behaviour is consistent with the information in the diagram. This means that a UML diagram is already making decisions w.r.t. the possible system structures quite early in the design process. The outcome diagram does not make such decisions.

In the rest of this section, we compare outcome diagrams to two UML diagrams, namely the state machine diagram and the component diagram.

\subsubsection{State Machine Diagram}

A state machine diagram is a finite state automaton. It defines the internal states of a system and the transitions between them. The state diagram captures the causality between the actions taken when the system changes states, but this does not map directly to the outcomes observed by an external user. There is however a relationship between a state diagram and an outcome diagram. An outcome can map to a sequence of state transitions; whereas, by examining the actions of a state diagram, it is possible to deduce the outcomes to expect from taking those actions.

\subsubsection{Block Diagram}

A block diagram specifies a system as a set of elements with their interconnections. We illustrate the difference between block diagrams and outcome diagrams using a simple example system, a user querying a front end that is connected to a database (Figure 5). The figure shows the refinement process: a system with an initially unknown structure is refined stepwise into a system that has a completely known structure. For the outcome diagram, the system performance can be obtained directly by composing the $\Delta$ Qs of the outcomes, using the rules described in Section 5. For the block diagram, it is harder to obtain system performance. This is because the block diagram does not define the expected outcomes of a system or their causality. The block diagram by itself does not have sufficient information to allow system performance to be calculated: we also need to know the expected outcome and the sequence of messages sent between blocks needed to achieve that outcome. As a final remark, the block diagram constrains the system structure to always have a front end and a database, whereas the outcome diagram is consistent with many alternative system structures. 


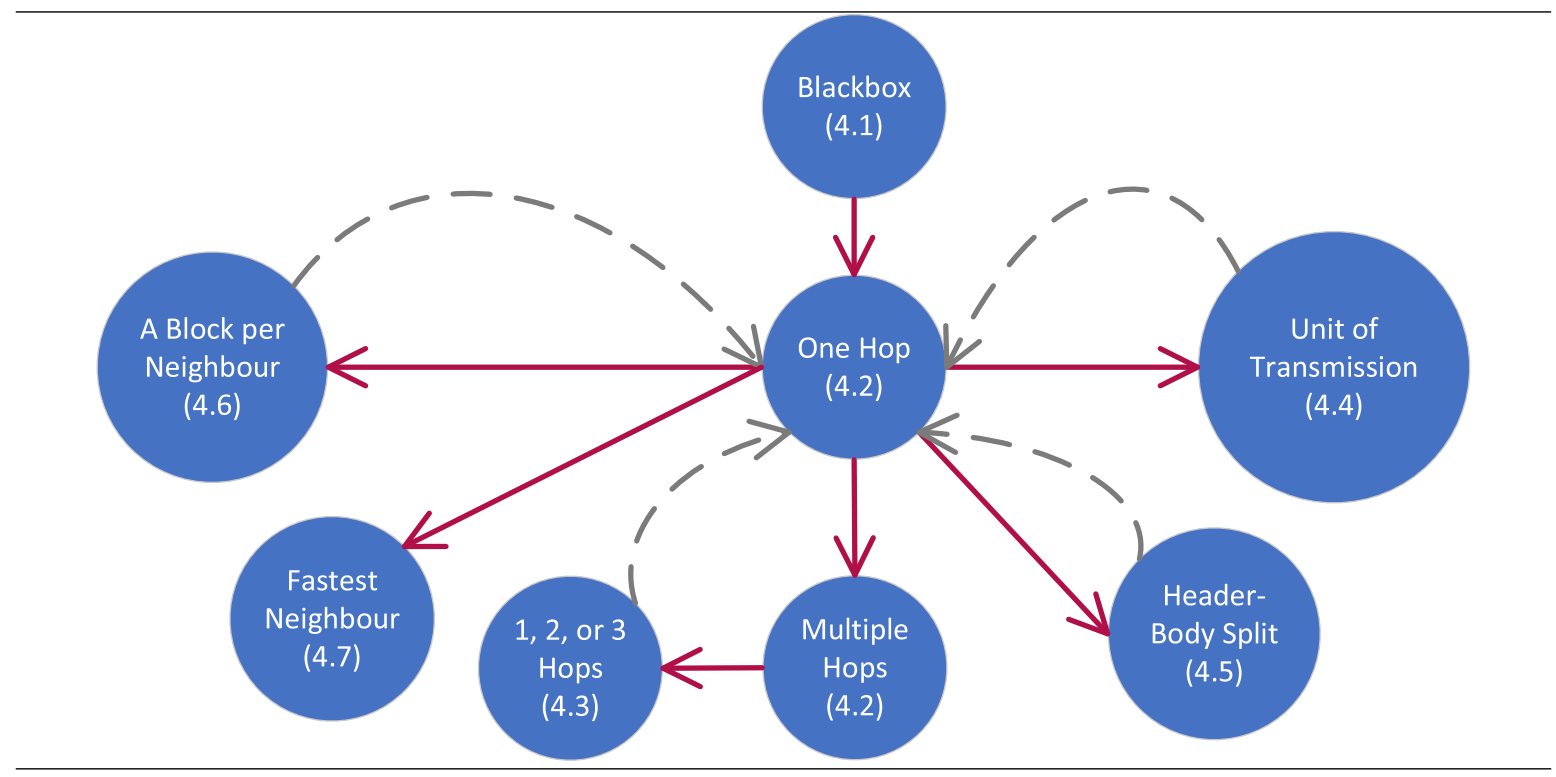

Figure 6. Design Exploration of a Blockchain Diffusion example, as described in Subsections 4.1-4.7. Solid lines are for refinement; dashed lines are for backtracking to coarser granularity.

\section{Design Exploration using Outcome Diagrams}

This section simulates how a design engineer could explore the blockchain diffusion example that was described in Section 2, using outcome diagrams. Figure 6 depicts that design exploration in the form of a threaded decision tree in the search space. Each node in the tree is an outcome diagram. Every node is labelled with a description plus the section in this paper where it is discussed. There are two types of edges: solid edges represent refinement steps (Definition 7); whilst dashed edges represent backtracks to take alternative directions of refinement. The formalism used in this section is presented in Section 5.

\subsection{Starting Off}

Initially, the design engineer knows almost nothing about the system. Perhaps, all they know is that there will be the following two observation locations:

- $A^{-}$: Block is ready to be transmitted by $A$.

- $Z^{+}$: Block is received and verified by $Z$.

The corresponding outcome diagram is

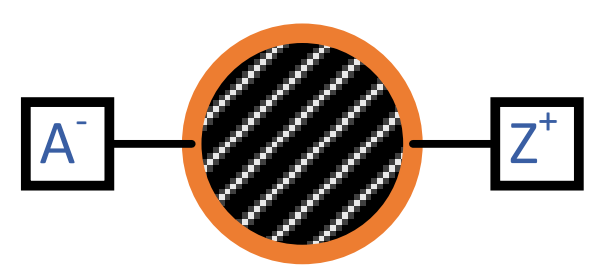

in which the only outcome is a black box. As will be detailed in Section 5 , the outcome expression to describe that outcome diagram is a $b$ ( $b$ for black boxes). 


\subsection{Early Analysis}

Given that the design engineer is not content with the current level of granularity, they wish to further detail the diagram by giving the black box a name, such as $o_{A \rightsquigarrow Z}$. In $\triangle Q S D$, we call adding that further detail a refinement. That refinement step is depicted below:

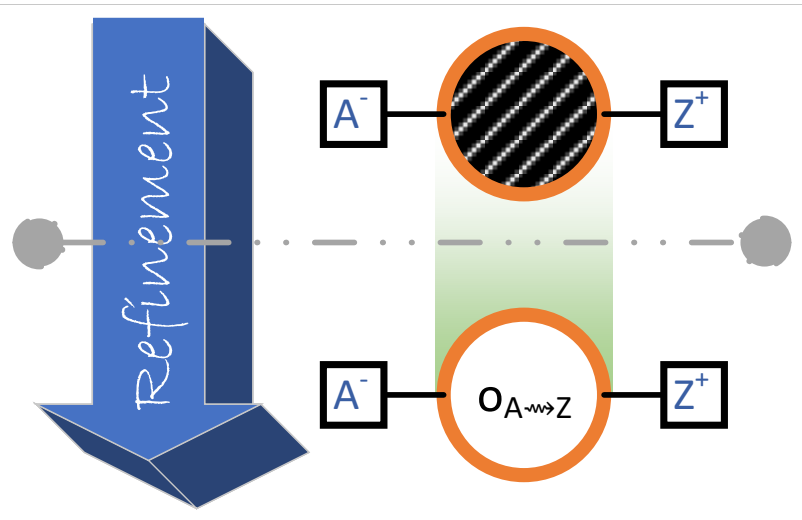

Here, the outcome diagram that is above the dashed line is refined into the one below. As will be discussed in Section 5, the (rewrite) rule that authorises this refinement is

$$
\mathcal{C}[b] \rightarrow \mathcal{C}[o]
$$

We call this rule (UNBX) for unboxing (a black box). The rule states that, in a context $\mathcal{C}$, a black box can be rewritten to any other outcome expression (but not to a black box). In this case, we choose the black box to be rewritten to an outcome variable called $o_{A \rightsquigarrow Z}$. This indicates the outcome of hopping directly from $A$ to $Z$.

Before producing more of our block diffusion algorithm's outcome diagram, we would like to take the time to apply some analysis. Refinements aside, suppose for a moment that there are two hops to make from $A$ to $Z$ : first from $A$ to an intermediate node $B$; and, then, from $B$ to $Z$. The corresponding outcome diagram for the two-hop journey from $A$ to $Z$ would then be:

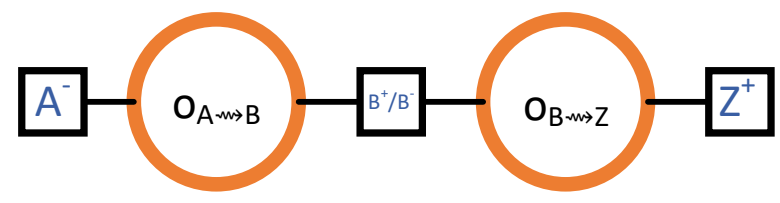

Here, $o_{A \rightsquigarrow B}$ and $o_{B \rightsquigarrow Z}$ are the outcomes of hopping from $A$ to $B$ and from $B$ to $Z$, respectively. Note also that the observation location between the above two outcomes is labelled $B^{+} / B^{-}$. That is because the observation $B^{+}$and $B^{-}$take place at the same location. For that reason, we will simply write $B$ to refer to that observation location. The same convention is used for similar intermediate locations. It is then easy to obtain the outcome diagram for three hops:

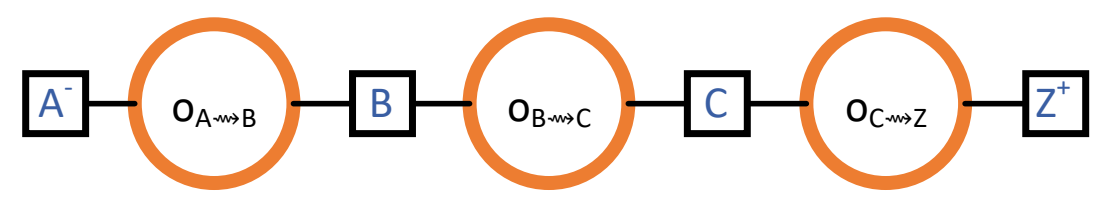

While outcome diagrams are visually more attractive; outcome expressions are algebraically more attractive. For example, the corresponding expression for two hops is $o_{A \rightsquigarrow B} \bullet \rightarrow \bullet o_{B \rightsquigarrow Z}$, where "• $\rightarrow \bullet$ " is the symbol we use for sequential composition: The sequential composition of $o_{A \rightsquigarrow B}$ and $o_{B} \rightsquigarrow Z$ is needed because the latter causally depends on the former. Likewise, the outcome expression for three hops is $o_{A \rightsquigarrow B} \bullet \bullet o_{B \rightsquigarrow C} \bullet \rightarrow o_{C \rightsquigarrow Z}$. Generalising that to $n$ hops then is easy: $o_{A \rightsquigarrow B_{1}} \bullet \bullet \bullet o_{B_{1} \rightsquigarrow B_{2}} \bullet \bullet \ldots \bullet \rightarrow \bullet$ $o_{B_{n} \rightsquigarrow Z}$, which we abbreviate as $o_{A \rightsquigarrow B_{1}} \bullet \bullet \bullet\left(: \bullet^{n-1} o_{B_{i} \rightsquigarrow B_{i+1}}\right) \bullet \bullet \bullet o_{B_{n} \rightsquigarrow Z}$. Parameterisation by $n$ hops 


\begin{tabular}{lr|rrrrrrrrrr}
\hline & & \multicolumn{2}{|c}{$64 \mathrm{kB}$} & \multicolumn{2}{c}{$256 \mathrm{kB}$} & \multicolumn{2}{c}{$512 \mathrm{kB}$} & \multicolumn{2}{c}{$1024 \mathrm{kB}$} & \multicolumn{2}{c}{$2048 \mathrm{kB}$} \\
Distance & time (s) & time(s) & RTTs & time(s) & RTTs & time(s) & RTTs & time(s) & RTTs & time(s) & RTTs \\
\hline Short & 0.012 & 0.024 & 1.95 & 0.047 & 3.81 & 0.066 & 5.41 & 0.078 & 6.36 & 0.085 & 6.98 \\
Medium & 0.069 & 0.143 & 2.07 & 0.271 & 3.94 & 0.332 & 4.82 & 0.404 & 5.87 & 0.469 & 6.81 \\
Lomg & 0.268 & 0.531 & 1.98 & 1.067 & 3.98 & 1.598 & 5.96 & 1.598 & 5.96 & 1.867 & 6.96 \\
\hline
\end{tabular}

Table 1. Representative times in seconds and round-trip-times (RTTs) for one-way TCP transmission of varying block sizes for short, medium and long distances between blockchain nodes.

is useful because it helps the design engineer determine the right $n$ for their blockchain. For example, a relevant question is: What is the optimal $n$ for block diffusion to be timely and for its load to be bearable? The formalisation in Section 5 instructs the design engineer as to how to achieve that and other goals. Before detailing the how, we take our moment to analyse a smaller example. Consider the two-hop scenario. Provided that the design engineer has $\Delta$ Qs for both $o_{A \rightsquigarrow B}$ and $o_{B \rightsquigarrow Z}$, they can use Definition 4 to work out the $\Delta \mathrm{Q}$ of $o_{A \rightsquigarrow B} \bullet \rightarrow \bullet o_{B \rightsquigarrow Z}$ :

$$
\Delta \mathrm{Q}\left(o_{A \rightsquigarrow B} \bullet \bullet o_{B \rightsquigarrow Z}\right)=\Delta \mathrm{Q}\left(o_{A \rightsquigarrow B}\right) \oplus \Delta \mathrm{Q}\left(o_{B \rightsquigarrow Z}\right) .
$$

In a similar vein, the design engineer can work out the $n$-hop scenario's $\Delta \mathrm{Q}$ for $n>1$ :

$$
\begin{aligned}
& \Delta \mathrm{Q}\left(o_{A \rightsquigarrow B_{1}} \bullet \bullet \bullet::_{i=1}^{n-1} o_{B_{i} \rightsquigarrow B_{i+1}}\right)\left.\bullet \bullet o_{B_{n} \rightsquigarrow Z}\right)= \\
& \Delta \mathrm{Q}\left(o_{A \rightsquigarrow B_{1}}\right) \oplus \bigoplus_{i=1}^{n-1} \Delta \mathrm{Q}\left(o_{B_{i} \rightsquigarrow B_{i+1}}\right) \oplus \Delta \mathrm{Q}\left(o_{B_{n} \rightsquigarrow Z}\right) .
\end{aligned}
$$

Then, using the formulation given in Definition 5, the design engineer can determine the constraints on $n$ that are needed in order for block diffusion to meet the overall timeliness requirements.

In practice, the time that is needed to transfer a block of data one hop depends on four main factors:

1. The size of the block;

2. The speed of the network interface;

3. The geographical distance of the hop (as measured by the time to deliver a single packet);

4. Congestion along the network path.

When we consider blockchain nodes that are located in data centres (which most block producers tend to be), the interface speed will typically be $1 \mathrm{~Gb} / \mathrm{s}$ or more. This is not a significant limiting factor for the systems of interest (see Section 5.4 for an analysis that explains this). In the setting that we are considering, congestion is generally minimal, and so this can also be ignored in the first instance. This leaves: i) block size, which we will take as a design parameter to be investigated later; and ii) distance, which we will consider now. For simplicity, we will consider three cases of geographical distance:

Short : the two nodes are located in the same data centre;

Medium : the two nodes are located in the same continent;

Long : the two nodes are located in different continents.

For pragmatic reasons, Cardano relies on the standard TCP protocol for data transfers. TCP transforms loss into additional delay, so the residual loss is negligible. At this point, we could descend into a detailed refinement of the TCP protocol, but equally we could simply take measurements; the compositionality of $\triangle \mathrm{QSD}$ means that it makes no difference where the underlying values come from. Table 1 shows measurements of the transit time of packets and the corresponding transfer time of blocks of various sizes, using hosts running on AWS data centre servers in Oregon, Virginia, London, Ireland and Sydney. Since we know that congestion is minimal in this setting, the spread of values will 


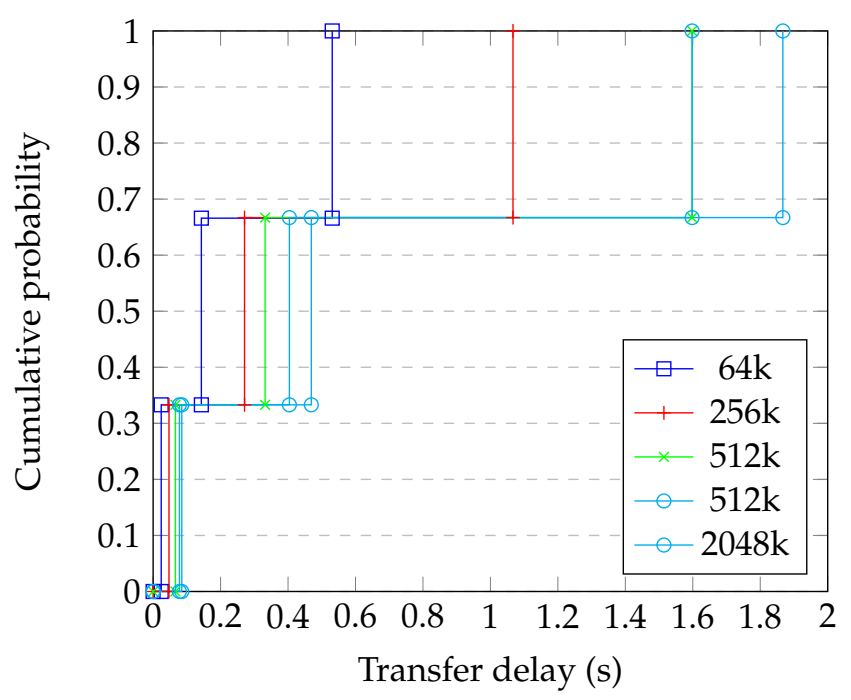

Figure 7. One-hop delay distributions per block size

be negligible, and so in this case the CDFs for the $\Delta$ Qs will be step functions. The transfer time for each block size is given both in seconds and in multiples of the basic round-trip time (RTT) between the hosts in question. Since the TCP protocol relies on the arrival of acknowledgements to permit the transmission of more data, it is unsurprising to see a broadly linear relationship, which could be confirmed by a more detailed refinement of the details of the protocol.

Given the randomness in the network structure and the selection of block-producing nodes, there remains some uncertainty on the length of an individual hop. At this point, we will assume that short, medium and long hops are equally likely, which we can think of as an equally-weighted probabilistic choice. In numerical terms, this becomes a weighted sum of the corresponding $\Delta Q$ s, as given in Table 1. This gives the distribution of transfer times per block size shown in figure 7 .

\subsection{Refinement and Probabilistic Choice}

Recall that $A$ and $Z$ are names for randomly chosen nodes, so the number of hops between $A$ and $Z$ is unkown. $\triangle Q S D$ tackles that uncertainty by offering an outcome diagram which involves probabilistic choice between the different number of hops that might be needed. Strictly speaking, a probabilistic choice is a binary operation. Hence, when there are more than two choices the outcome diagram will cascade probabilistic choices. In the general formulation, there are at most $n$ hops. In order to produce that, the design engineer exercises a step-by-step refinement of the single-hop outcome diagram. The first refinement introduces the choice between one or two hops: 


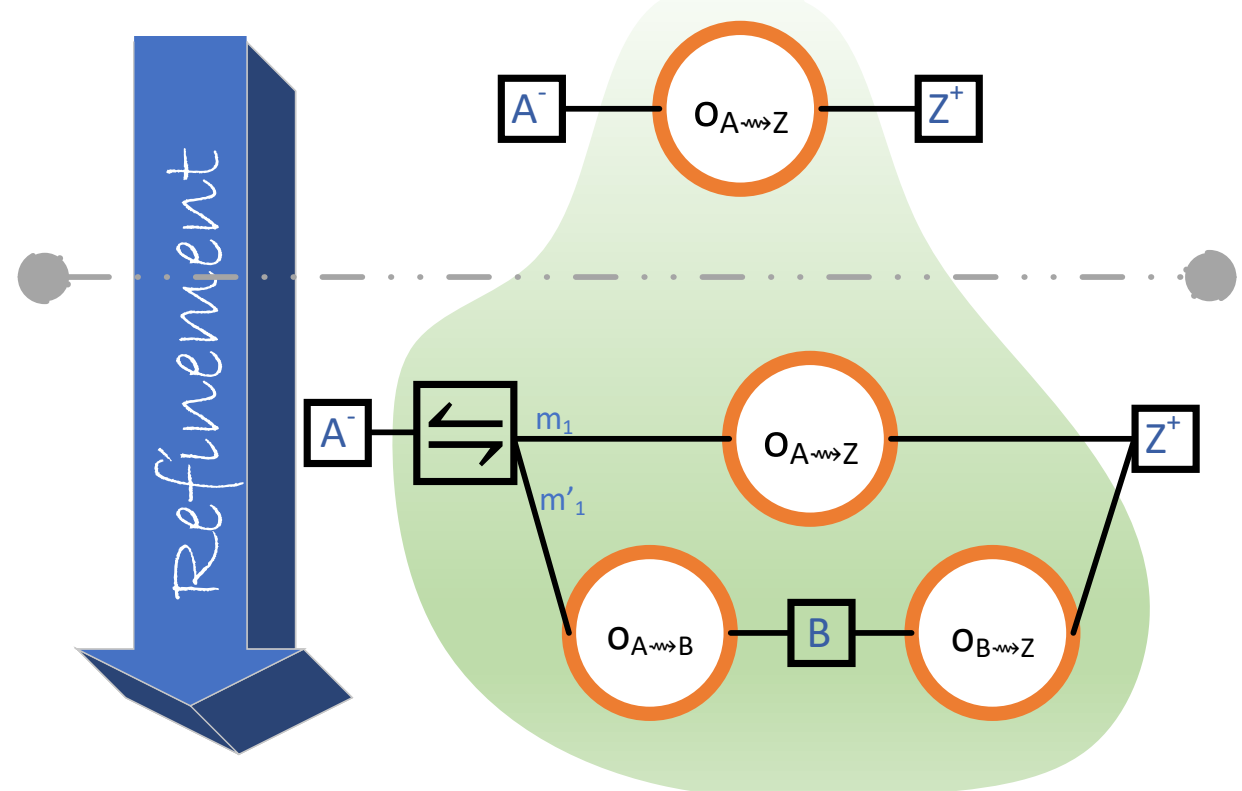

The equivalent outcome expression is $o_{A \rightsquigarrow Z} \stackrel{\frac{m_{1}}{m_{1}^{\prime}}}{(}\left(o_{A \rightsquigarrow B} \bullet \succ \bullet o_{B \rightsquigarrow Z}\right)$, a probabilistic choice between one or two hops with respective weights $m_{1}$ and $m_{1}^{\prime}$. The corresponding (rewrite) rule is:

$$
\mathcal{C}[o] \rightarrow \mathcal{C}\left[o^{\prime} \underset{m^{\prime \prime}}{\stackrel{m^{\prime}}{=}} o^{\prime \prime}\right]
$$

which we call (PROB) (for probabilistic choice). Here is how we applied (РROB) to arrive from the single hop to the probabilistic choice between one hop and two hops:

$$
o_{A \rightsquigarrow Z} \rightarrow o_{A \rightsquigarrow Z} \stackrel{\frac{m_{1}}{m_{1}^{\prime}}}{(}\left(o_{A \rightsquigarrow B} \bullet \rightarrow o_{B \rightsquigarrow Z}\right)
$$

That is, $\mathcal{C}$ in the above refinement is an empty context.

Next, the design engineer further refines the two-hop part to the probabilistic choice between two or three hops. 


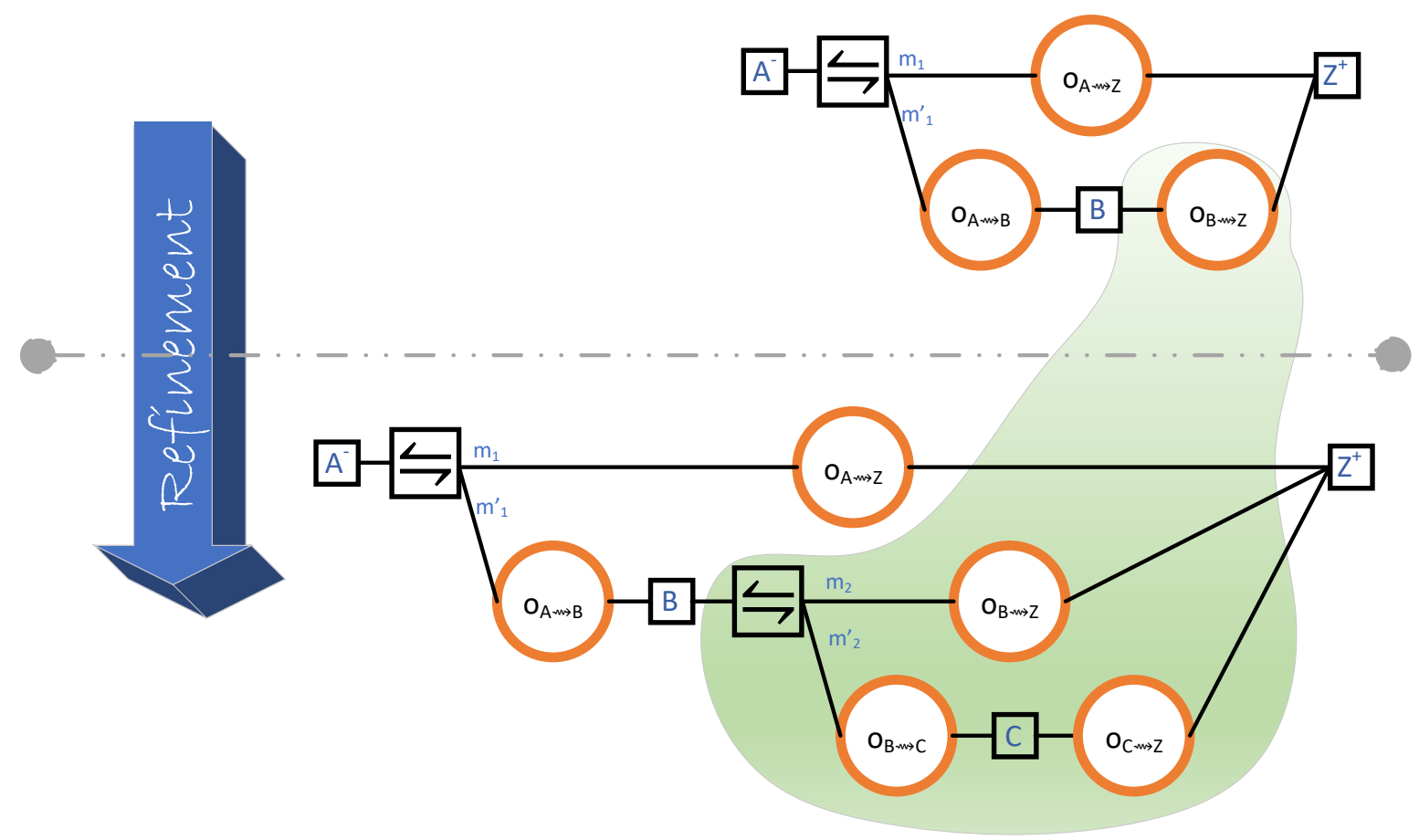

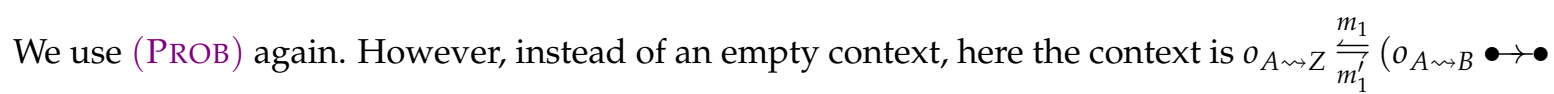
$[].):$

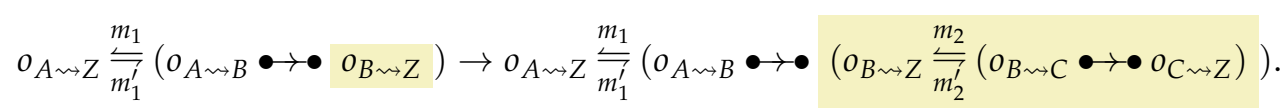

The design engineer can continue refinement until a predetermined number of hops is reached. Alternatively, they can keep the number of hops as a parameter and analyse the corresponding parameterised outcome expression for timeliness, behaviour under load, etc.

Figure 8 shows the result of applying equation 2 to the sequence of outcome expressions corresponding to one, two, ... five sequential hops using the transfer delay distribution shown in Figure 7 , for a $64 \mathrm{kB}$ block size. It can be seen that there is a $95 \%$ probability of the block arriving within 2s. In contrast, Figure 9 shows the corresponding sequence of delay distributions for a $1024 \mathrm{kB}$ block size, where the 95th percentile of transfer time is more than $5 \mathrm{~s}$.

If we know the distribution of expected path lengths, we can combine the $\Delta$ Qs for different hop counts using (PROB). Table 2 shows the distribution of paths lengths in simulated random graphs having 2500 nodes and a variety of node degrees [18]. Using the path length distribution for nodes of degree 10, for example, then gives the transfers delay distribution shown in Figure 10.

\begin{tabular}{c|rrrr}
\hline Length & \multicolumn{5}{|c}{ Node degree } \\
& \multicolumn{1}{|c}{5} & \multicolumn{1}{c}{10} & \multicolumn{1}{c}{15} & \multicolumn{1}{c}{20} \\
\hline 1 & 0.20 & 0.40 & 0.60 & 0.80 \\
2 & 1.00 & 3.91 & 8.58 & 14.72 \\
3 & 4.83 & 31.06 & 65.86 & 80.08 \\
4 & 20.18 & 61.85 & 24.95 & 4.40 \\
5 & 47.14 & 2.78 & 0.00 & \\
6 & 24.77 & 0.00 & & \\
7 & 1.83 & & & \\
8 & 0.05 & & & \\
\hline
\end{tabular}

Table 2. Percentage of paths having a given length in a random graph of 2500 nodes of varying degree 


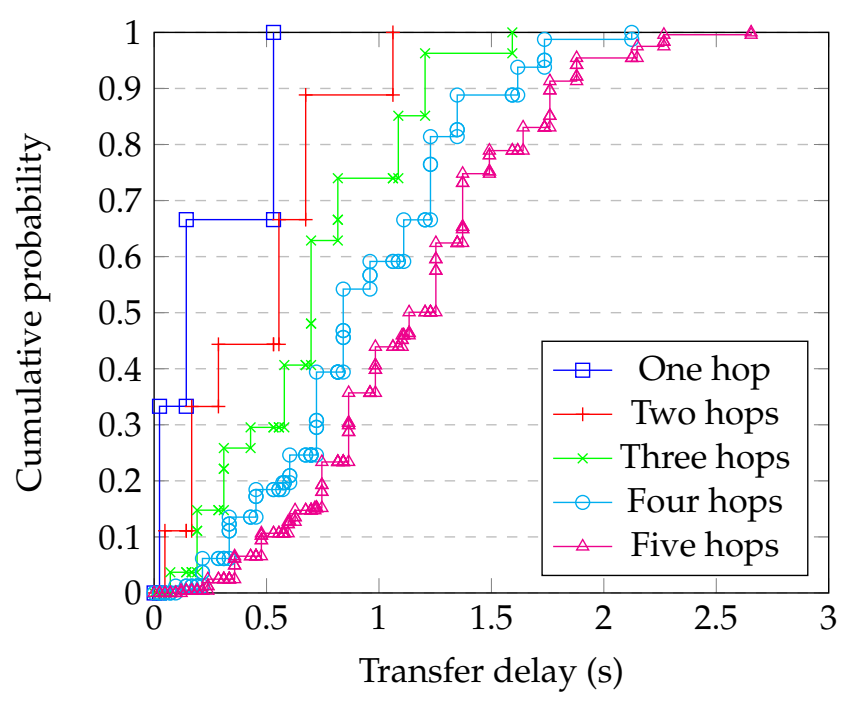

Figure 8. Multi-hop delay distributions for $64 \mathrm{k}$ block size

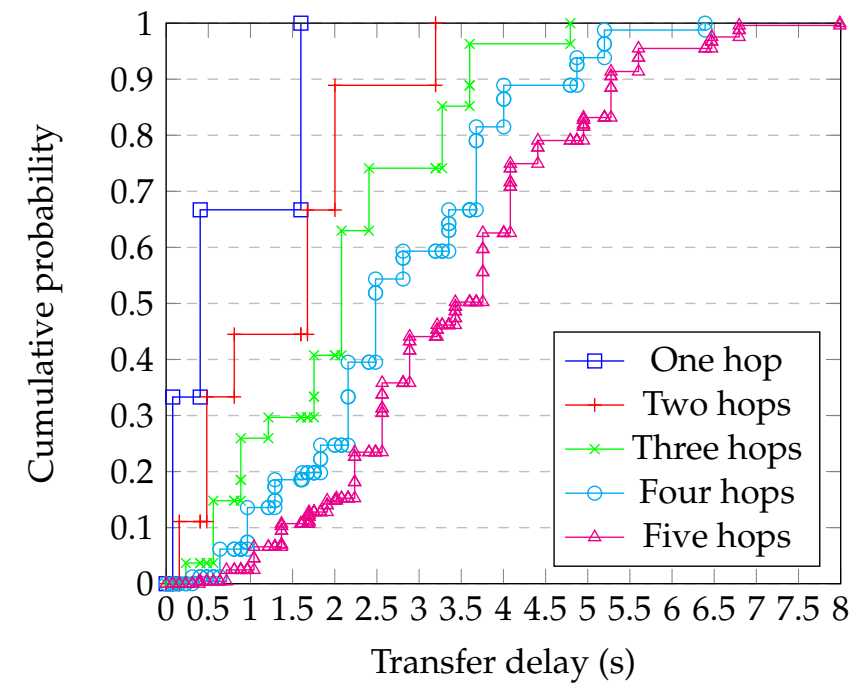

Figure 9. Multi-hop delay distributions for 1024k block size

Alternative Refinements

Suppose that, instead of investigating the number of hops, the design engineer is now interested in studying the steps within a single hop. There are various ways to do this. In Sections 4.4-4.7, we will consider four different ways that can be used when $A$ and $Z$ are neighbours. each of which refines $o_{A \rightsquigarrow Z}$. These refinements are all instances of the (ELAB) (rewrite) rule (for elaboration):

$$
\mathcal{C}\left[o_{v}\right] \rightarrow \mathcal{C}[o]
$$

The following sections are also important for another reason. So far, we have traversed the threaded tree of refinement in a depth-first way; the upcoming subsections traverse that tree in a breadth-first way. $\triangle Q S D$ allows the design engineer to choose between depth-first and breadth-first refinement at any point in their design exploration. 


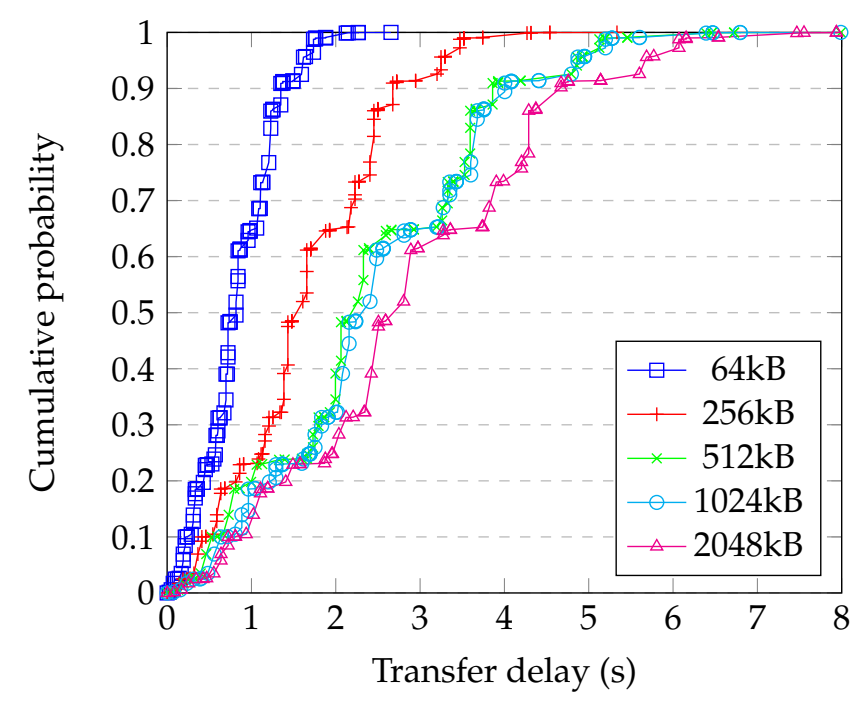

Figure 10. Multi-hop delay distributions for varying block size in a graph of 2500 nodes with node degree 10

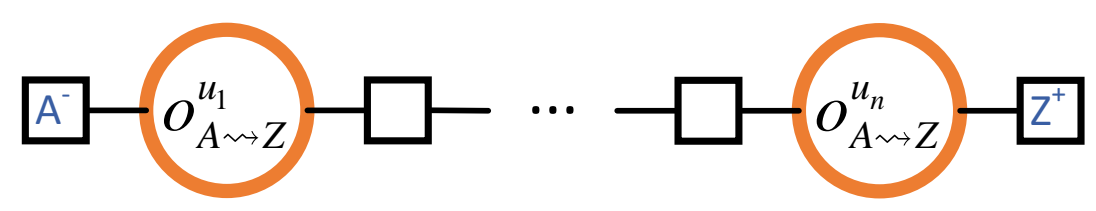

Figure 11. Breaking down the Transmission of a Message into $n$ Smaller Units.

\subsection{Breaking Down Transmissions into Smaller Units}

Network transmissions are typically broken down into the transmission of smaller units. Depending on the layering of the network protocols, that might, for example mean dividing a high-level message into several smaller packets. In a similar vein, the design engineer might decide to study block diffusion in terms of smaller units of transmission. For example, they might want to study the division of $o_{A \rightsquigarrow Z}$ into $n$ smaller unit operations $o_{A \rightsquigarrow Z}^{u_{1}}, \ldots, o_{A \rightsquigarrow Z}^{u_{n}}$. The resulting outcome diagram is shown in Figure 11. The corresponding outcome expression would then be $o_{A \rightsquigarrow Z} \rightarrow o_{A \rightsquigarrow Z}^{u_{1}} \bullet \succ \bullet \ldots \bullet \bullet o_{A \rightsquigarrow Z^{\prime}}^{u_{n}}$ which we abbreviate as $o_{A \rightsquigarrow Z} \rightarrow: o_{A \rightsquigarrow Z}$. This refinement can happen at different levels of granularity and is fairly repetitive. However, this is the level at which details of the transmission protocol such as TCP could be introduced if required.

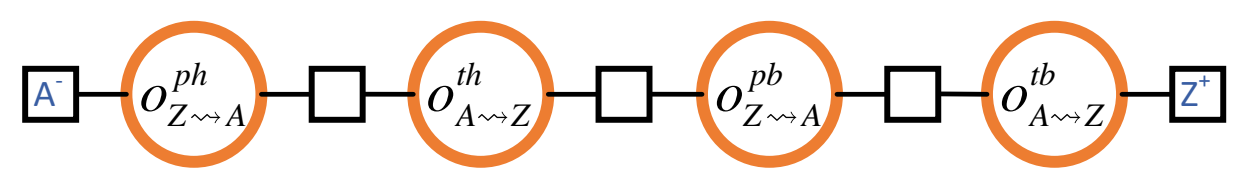

Figure 12. Splitting a Block Transmission into its Constituent Parts: Header $(p h / t h)$ and Body $(p b / t b)$. 


\subsection{Header-Body Split}

In Cardano Shelley, an individual block transmission involves a dialogue between a sender node, $A$, and a recipient node, $Z$. We represent the overall transmission as $o_{A \rightsquigarrow Z}$. This can be refined into the following sequence:

1. Permission for Header Transmission $\left(o_{Z \rightsquigarrow A}^{p h}\right)$ : Node $Z$ grants the permission to node $A$ to send it a header.

2. Transmission of the Header $\left(o_{A \rightsquigarrow Z}^{\text {th }}\right)$ : Node $A$ sends a header to node $Z$.

3. Permission to for Body Transmission $\left(o_{Z \rightsquigarrow A}^{p b}\right)$ : Node $Z$ analyses the header that was previously sent to it by $A$. Once the suitability of the block is determined via the header, node $Z$ grants permission to $A$ to send it the respective body of the previously sent header.

4. Transmission of the Body $\left(o_{A \rightsquigarrow Z}^{t b}\right)$ : Finally, $A$ sends the block body to $Z$.

The motivation for the header/body split and the consequential dialogue is optimisation of transmission costs. Headers are designed to be affordably cheap to transmit. In addition, they carry enough information about the body to enable the recipient to verify its suitability. The body is only sent once the recipient has done this. This prevents the unnecessary transmission of block bodies when they are not required. Since bodies are typically many orders of magnitude larger than headers, considerable network bandwidth can be saved in this way. Moreover, the upstream node is not permitted to send another header until given permission to do so by the downstream node, in order to prevent a denial-of-service attack in which a node is bombarded with fake headers, so this approach also reduces latency when bodies are rejected. In practice, the first permission is sent when the connection between peers is established, and the permission renewed immediately after the header is received, so that the upstream peer does not have to wait unnecessarily. The design engineer can therefore refine $o_{A \rightsquigarrow Z}$ into the finer grained outcomes shown in Figure 12. The corresponding outcome expression is $o_{Z \rightsquigarrow A}^{p h} \bullet>o_{A \rightsquigarrow Z}^{t h} \bullet>o_{Z \rightsquigarrow A}^{p b} \bullet \rightarrow o_{A \rightsquigarrow Z}^{t b}$.

Note that the protocol described here is between directly connected neighbours - these requests are not forwarded to other nodes. This is thus a refinement of the one-hop block transfer process. The significance of this refinement is that it shows that an individual outcome that, at a given level of granularity, is unidirectional (i.e., only from one entity in the system to another) might, at a lower level of granularity, very well be a multi-directional conversation.

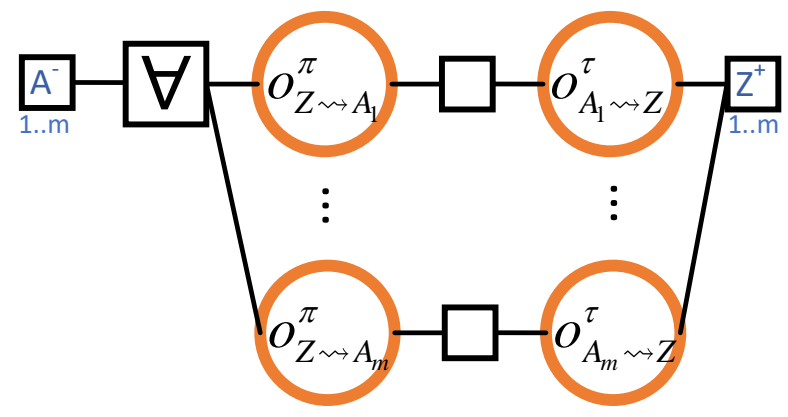

Figure 13. Obtaining one Block from each Neighbour when Rejoining the Blockchain.

\subsection{Obtaining one Block from each Neighbour when Rejoining the Blockchain}

Consider the situation where a node $Z$ rejoins the blockchain after being disconnected for some period of time. $Z$ will be out-of-date w.r.t. the recently generated blocks and will need to update itself. Let us consider the lucky situation where $Z$ can acquire all the blocks that it is missing from its 
neighbours; that is, it can acquire the blocks with only one hop but from different neighbours. For demonstration purposes, we now make a number of simplifying assumptions:

- Upon its return to the blockchain, $Z$ is $m$ blocks behind, where $m$ is less than or equal to the number of $Z$ 's neighbours.

- Each neighbour $A_{i}$ of $Z$ transmits precisely one block to $Z$.

- The header-body split refinement of Section 4.5 is not considered. Therefore, there are only two steps (instead of the actual four):

1. $o_{Z \rightsquigarrow A_{i}}^{\pi}$ for when $Z$ grants permission to $A_{i}$. And,

2. $o_{Z \rightsquigarrow A_{i}}^{\tau}$ for when $A_{i}$ transmits the (entire) block to $Z$.

With those simplifications in place, the outcome diagram will be as shown in Figure 13. This shows that $Z$ will be up-to-date when all the $m$ (selected) neighbours of its are granted permission and have finished sending their blocks to $Z$. Note that the outcome diagram has, in fact, $m$ starting observation locations and $m$ terminating observation locations. This is the reason for the 1..m notation immediately below each of those observation locations. The corresponding outcome expression is

$$
\forall\left(\left(o_{Z \rightsquigarrow A_{1}}^{\pi} \bullet \rightarrow \bullet o_{A_{1} \rightsquigarrow Z}^{\tau}\right)\|\cdots\|\left(o_{Z \rightsquigarrow A_{m}}^{\pi} \bullet \rightarrow \bullet o_{A_{m} \rightsquigarrow Z}^{\tau}\right)\right)
$$

which we abbreviate as $\|^{\forall}\left(o_{Z \rightsquigarrow A_{i}}^{\pi} \bullet \rightarrow o_{A_{i} \rightsquigarrow Z}^{\tau}\right)_{1}^{m}$.

One reason why this refinement is particularly interesting is that it allows an easy demonstration of our load analysis from Section 5.4. Fix a resource $\rho$ such as network capacity. Pick a time $t$ between the first observation made at an $A_{i}^{-}$and the last observation made at a $Z_{i}^{+}$. According to Definition 10, the static amount of work $S$ at time $t$ that is required for performing $\|^{\forall}\left(o_{Z \rightsquigarrow A_{i}}^{\pi} \bullet \bullet \bullet o_{A_{i} \rightsquigarrow Z}^{\tau}\right)_{1}^{m}$ is the sum of the static amounts of work $S$ that is required at $t$ for performing each $o_{Z \rightsquigarrow A_{i}}^{\pi} \bullet \rightarrow \bullet o_{A_{i} \rightsquigarrow Z}^{\tau}$ (where $1 \leq i \leq m)$ :

$$
S\left[\|\|^{\forall}\left(o_{Z \rightsquigarrow A_{i}}^{\pi} \bullet \rightarrow \bullet o_{A_{i} \rightsquigarrow Z}^{\tau}\right)_{i=1}^{m} \rrbracket(t)=\sum_{i=1}^{m} S\left[o_{Z \rightsquigarrow A_{i}}^{\pi} \bullet \rightarrow \bullet o_{A_{i} \rightsquigarrow Z}^{\tau}\right](t) .\right.
$$

Equation (3) describes an approach to aggregating offered load on a resource. Consider an ephemeral resource - such as a communications network interface - a design interest might be to understand the intensity of use of this interface. For example, for a design requirement to be (at this level of detail) feasible, the average use of the interface has to be less than its capacity. This is the basic precondition for the demand on the resource to possess a feasible schedule. The RHS of equation (3) captures this process as a piece-wise summation of the load intensities. Building on the time to transfer blocks (Table 1), and noting (from Section 2.1) that the body of a block is forwarded in response to a request (which takes one round trip time); the total block volume is delivered in the total time minus the round trip time. For the 'Near' peers shipping a $64 \mathrm{kB}$ block, this means an intensity of $42.7 \mathrm{Mb} / \mathrm{s}(8 * 64 e 3 /(0.024-0.012))$ before incorporating any other network related overheads (such as layered headers). Table 3 captures that load intensity approximation.

\begin{tabular}{l|rrrrr}
\hline & \multicolumn{5}{|c}{ Block size (kB) } \\
Distance & 64 & 256 & 512 & 1,024 & 2,048 \\
\hline Short & 42.7 & 58.5 & 75.9 & 151.7 & 224.4 \\
Medium & 6.9 & 10.1 & 15.6 & 31.1 & 41.0 \\
Long & 1.9 & 2.6 & 3.1 & 6.2 & 10.2 \\
\hline
\end{tabular}

Table 3. Average load intensities (in Mbit/s) implied by time to load from Table 1

This provides an insight into the likely capacity constraints for differing degrees of connectivity and, by inference, an insight into the system level design trades. From Tables 1 and 3 it can be seen that smaller 
geographic distribution can lead to lower forwarding times assuming that (for a fixed communications capacity) the number of associating peers is suitably reduced. Assessments such as this give a measure of the likely "slack" in the design; those portions of the design that have less "slack" represent design elements that might need more detailed refinement and/or other strategies to ensure their feasibility. Note that a dedicated support tool for $\triangle$ QSD would easily be able to manipulate these complex outcome diagrams, giving a formally correct analysis, with very little mental burden for the design engineer.

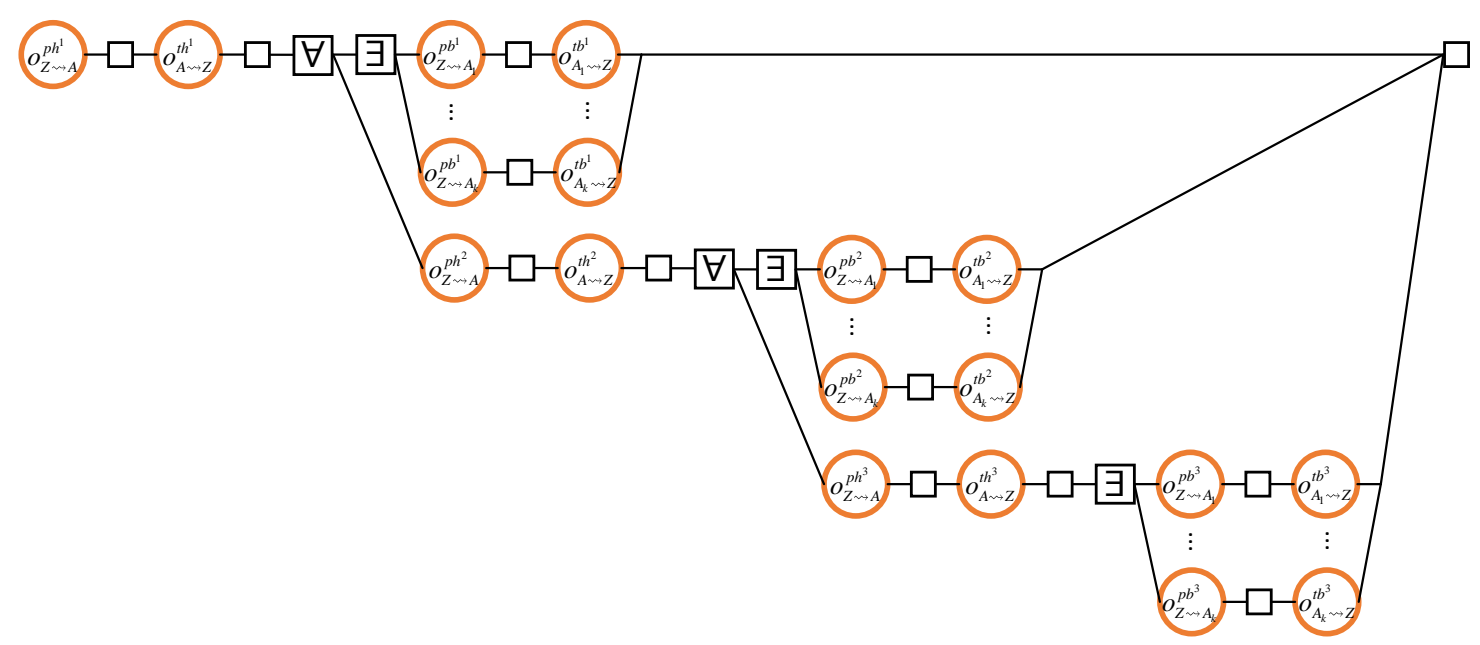

Figure 14. Obtaining a Block from the Fastest Neighbour.

\subsection{Obtaining a Block from the Fastest Neighbour}

Section 4.5 discussed splitting the header and body for optimisation reasons. One assumption in that design is that the header and the body will be taken from the same neighbour. It turns out that this assumption will not necessarily lead to the fastest solution. In fact, when $Z$ determines that it is interested in a block that it has received the header of, it may obtain it from any of its neighbours that have signalled that they have it. In particular, Cardano nodes keep a record of the $\Delta$ Qs of their neighbours' block delivery. This allows them to obtain bodies from their fastest neighbour(s). In other words, once a node determines the desirability of a block (via its header), it is free to choose to take the body from any of its neighbours that have provided the corresponding header. As long as only timeliness is a concern - and not when resource consumption is also of interest - a race can occur between all neighbours, with the fastest neighbour winning the race. The diagrams in this section assume such a race.

Now, like Section 4.6, consider the situation where $Z$ reconnects to the blockchain after being disconnected for some time. Our design in Section 4.6 assumes that there is no causality between the $m$ blocks that $Z$ needs to obtain. In reality, that is not correct: there is a causal order between those blocks, and that order can be rather tricky to define; it might take a couple of reads before the matter is fully digested. There are two separate total orders between blocks:

CO1. For each block, the header must be transmitted before the body (so that the recipient node can determine the suitability of the block before the body transmission);

CO2. Headers of the older blocks need to be transmitted before those of the younger blocks (note, however, that there is no causal relationship between the body transmissions).

This section considers the situation when the design engineer investigates the above race as well as $\mathrm{CO} 1$ and $\mathrm{CO} 2$. Suppose that once $\mathrm{Z}$ reconnects to the blockchain, it is exactly $m=3$ blocks behind the 
current block. Suppose also that $Z$ has $k$ neighbours. The corresponding outcome diagram is shown in Figure 14. The fork that is causally dependent on $o_{A \rightsquigarrow Z}^{t h^{3}}$ is done when any of its prongs is done, that is, as soon as any neighbour of $Z$ has finished transmitting the third block to $Z$. The other " $\exists$ " forks are similar.

The corresponding outcome expression is:

$$
\begin{aligned}
& o_{Z \rightsquigarrow A}^{p h^{1}} \bullet \longrightarrow o_{A \rightsquigarrow Z}^{t h^{1}} \bullet \rightarrow \bullet \forall\left(\exists\left[\left(o_{Z \rightsquigarrow A_{1}}^{p b^{1}} \bullet \rightarrow o_{A_{1} \rightsquigarrow Z}^{t b^{1}}\right)\|\cdots\|\left(o_{Z \rightsquigarrow A_{k}}^{p b^{1}} \bullet \bullet \bullet o_{A_{k} \rightsquigarrow Z}^{t b^{1}}\right)\right] \|\right. \\
& \left(o _ { Z \rightsquigarrow A } ^ { p h ^ { 2 } } \bullet \bullet o _ { A \rightsquigarrow Z } ^ { t h ^ { 2 } } \bullet \bullet \forall \left(\exists\left[\left(o_{Z \rightsquigarrow A_{1}}^{p b^{2}} \bullet \bullet o_{A_{1} \rightsquigarrow Z}^{t b^{2}}\right)\|\cdots\|\left(o_{Z \rightsquigarrow A_{k}}^{p b^{2}} \bullet \bullet o_{A_{k} \rightsquigarrow Z}^{t b^{2}}\right)\right] \|\right.\right. \\
& \left.\left.\left(o_{Z \rightsquigarrow A}^{p h^{3}} \bullet \bullet o_{A \rightsquigarrow Z}^{t h^{3}} \bullet \bullet \bullet\left[\left(o_{Z \rightsquigarrow A_{1}}^{p b^{3}} \bullet \bullet o_{A_{1} \rightsquigarrow Z}^{t b^{3}}\right)\|\cdots\|\left(o_{Z \rightsquigarrow A_{k}}^{p b^{3}} \bullet \bullet o_{A_{k} \rightsquigarrow Z}^{t b^{3}}\right)\right]\right)\right)\right) \text { ). }
\end{aligned}
$$

We would like to invite the reader to take their time to pair the above diagram with our explanations above. We understand that the diagram and to a greater degree the expression can look impenetrable. Compositionality of our formalism (inherited from that of $\triangle \mathrm{QSD}$ ) comes to rescue! Indeed, we can observe that the race pattern is rather repetitive. Thus, we can wrap the entire race into three new outcomes $o_{. \rightsquigarrow Z}^{b^{1}}, o_{. \rightsquigarrow Z}^{b^{2}}$, and $o_{. \rightsquigarrow Z}^{b^{3}}$. The intention is for $o_{. \rightsquigarrow Z}^{b^{1}}$, for example, to be the outcome of obtaining the first body transmitted to $Z$ by any one of its $k$ neighbours (that is, we are using "." in the subscript of $o_{. \rightsquigarrow Z}^{b^{1}}$ as a wildcard). This makes the outcome diagram considerably simpler:

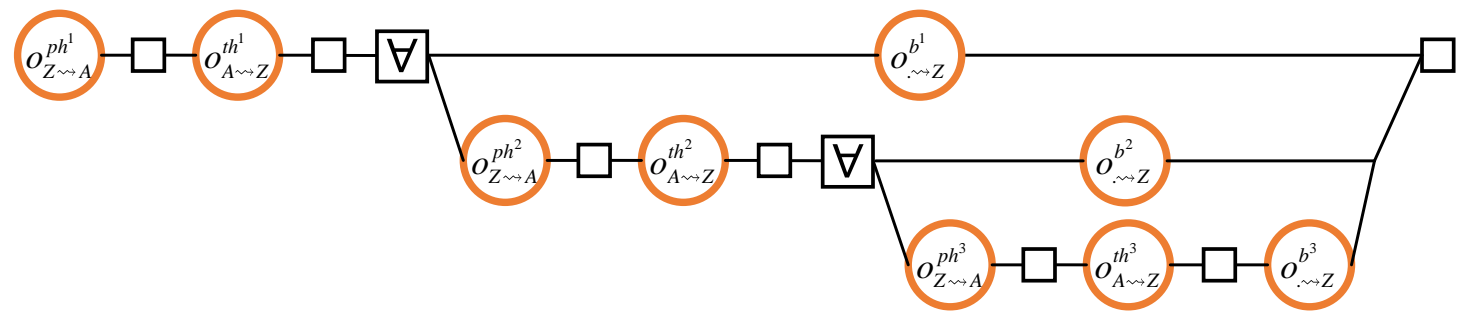

where

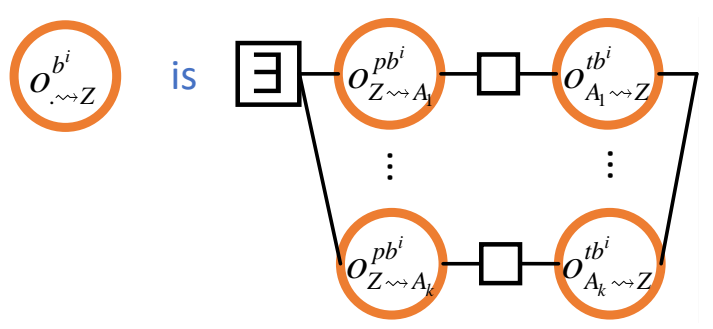

These new diagrams make it easy to spot the lack of causal relationship between the $o_{. \sim Z}^{b^{i}} \mathrm{~s}$. Hence, there is no causal order between the body transmission despite the existence of CO1 and CO2. The corresponding outcome expression also becomes considerably simpler:

$$
o_{Z \rightsquigarrow A}^{p h^{1}} \bullet \rightarrow \bullet o_{A \rightsquigarrow Z}^{t h^{1}} \bullet \rightarrow \bullet \forall\left(o_{. \rightsquigarrow Z}^{b^{1}} \|\left(o_{Z \rightsquigarrow A}^{p h^{2}} \bullet \rightarrow \bullet o_{A \rightsquigarrow Z}^{t h^{2}} \bullet \rightarrow \bullet \forall\left(o_{. \rightsquigarrow Z}^{b^{2}} \|\left(o_{Z \rightsquigarrow A}^{p h^{3}} \bullet \rightarrow o_{A \rightsquigarrow Z}^{t h^{3}} \bullet \rightarrow \bullet o_{. \rightsquigarrow Z}^{b^{3}}\right)\right)\right)\right)
$$

where

$$
o_{. \rightsquigarrow Z}^{b^{i}}=\exists\left[\left(o_{Z \rightsquigarrow A_{1}}^{p b^{i}} \bullet \rightarrow \bullet o_{A_{1} \rightsquigarrow Z}^{t b^{i}}\right)\|\cdots\|\left(o_{Z \rightsquigarrow A_{k}}^{p b^{i}} \bullet \rightarrow \bullet o_{A_{k} \rightsquigarrow Z}^{t b^{i}}\right)\right]
$$

which we abbreviate as

$$
\|^{\exists}\left(o_{Z \rightsquigarrow A_{j}}^{p b^{i}} \bullet \rightarrow \bullet o_{A_{j} \rightsquigarrow Z}^{t b^{i}}\right)_{j=1}^{m} \cdot
$$

The latter outcome diagrams and outcome expressions are now relatively easy to follow. 


\subsection{Summary}

The refinements and analysis in this section have captured part of the design journey of Cardano Shelley. In Section 4.1, we defined a 'top level' outcome of interest, that of diffusing a block from an arbitrary source node to an arbitrary destination in a bounded time and with bounded resource consumption. In Section 4.2 we refined this to examine the implications of forwarding the block through a sequence of intermediate nodes, and in Section 4.3 we factored in the expected distribution of path lengths. This allows an exploration of the trade-offs between graph size, node degree, block size and diffusion time.

In Section 4.4 we showed how $\triangle Q S D$ can be used to explore orthogonal aspects of the design, in this case how blocks of data are in fact transmitted as a sequence of packets. This could be extended into a full analysis of some transmission protocol such as TCP or QUIC. In Section 4.5 we analysed the effects of splitting blocks into a header and a body in order to reduce resource consumption, and in Section 4.6 the potential for speeding up block downloading by using multiple peers in parallel. An analysis of the network resource consumption in this case gave a flavour of how the $\Delta$ QSD paradigm encompasses resource as well as timeliness constraints.

In Section 4.7 we discussed how $\Delta \mathrm{Q}$ is used in Cardano Shelley in operation as well as in design, to optimise the choice of peer from which to obtain a block.

All of this, together with further optimisations such as controlling the formation of the node graph to achieve a balance between fast block diffusion and resilience to partitioning, has produced an industry-leading blockchain implementation that functions reliably delivering a block up to $72 \mathrm{kB}$ every 20s on average. A snapshot of the 90th centile of block diffusion times over nearly 48 hours is given in Figure 15.

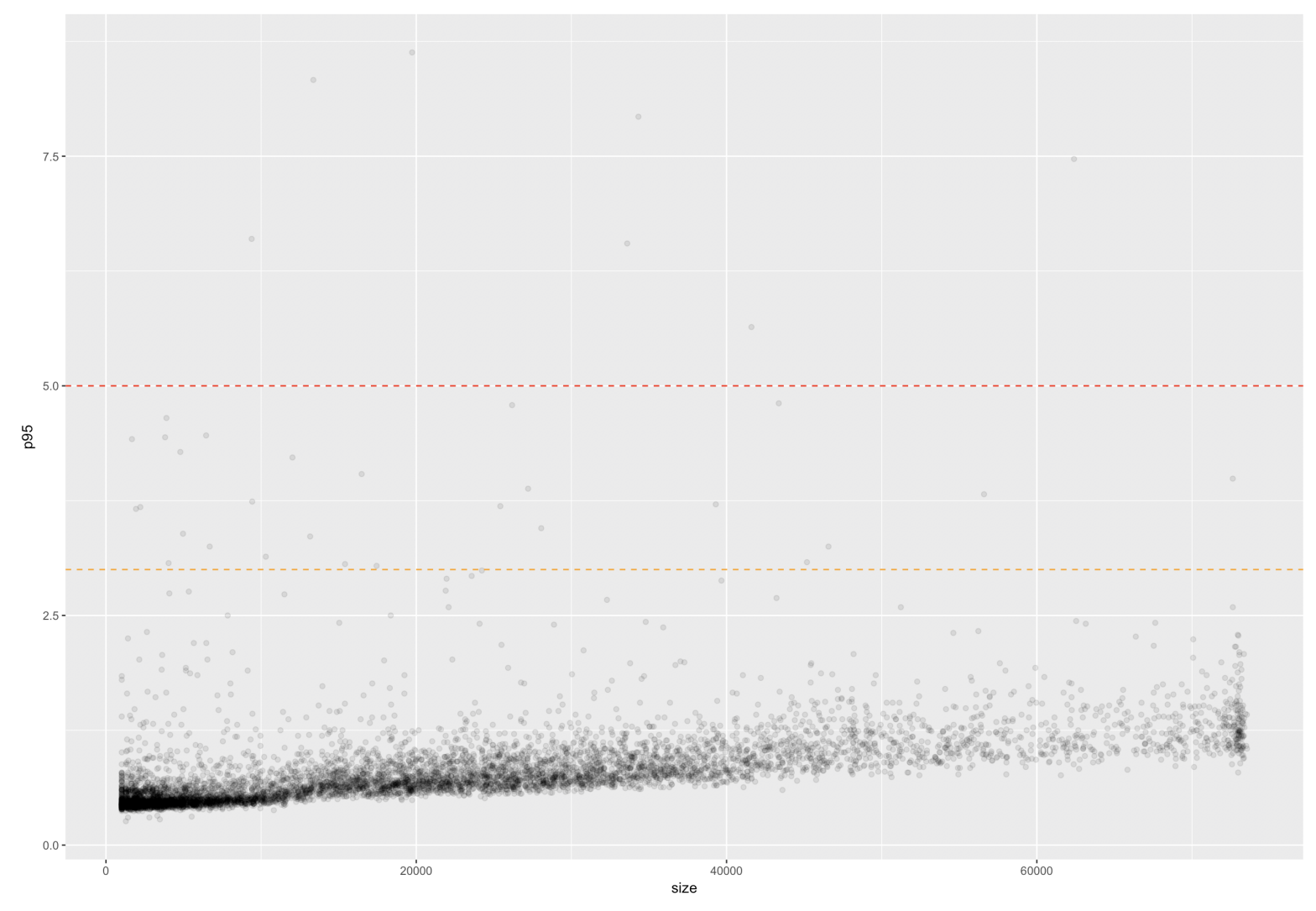

Figure 15. 95th centile of block diffusion times as a function of block size (in seconds).

Work is ongoing to further improve the performance of Cardano, based securely in the $\triangle$ QSD paradigm. 


\section{A Formalisation of $\Delta Q S D$}

The examples that were presented in Section 4 all build on the formalisms that we will present in this section. We start by describing the notational conventions that we will use here (Section 5.1). We then provide the syntax (Definition 1) for outcome expressions and formalise the rewrite rules that define the valid transitions between possible outcomes (Definition 3). In Sections 5.3 and 5.4, we provide corresponding denotational semantics for both timeliness and load. These provide the bases for constructing formal timeliness and load analyses that can be used as part of $\triangle Q S D$. The analyses have so far been deployed manually to inform design decisions for a number of complex real-world systems. Our longer-term intention is that they should be implemented as part of a design exploration toolset that will support $\triangle \mathrm{QSD}$. Additional semantics and analyses are also possible, of course, and could be used to support alternative design explorations or to provide further details about timeliness, load etc.

\subsection{Notational Conventions}

Let $\mathbb{A}, \mathbb{B}, \mathbb{C}, \ldots$ range over sets of values, and let lower case letters, $a, b, c, \ldots$ range over elements of those sets. For predicates, we write pred $(x)$.

\subsection{Syntax}

Let $\mathbb{B} \ni b$ and $\mathbb{O}_{v} \ni o_{v}$. We refer to black boxes and outcome variables together as base variables: $\overline{\mathbb{B}}=\mathbb{O}_{v} \cup \mathbb{B}$, where $\overline{\mathbb{B}} \ni \beta$.

Definition 1. The abstract syntax of outcome expressions is:

$$
\begin{array}{rlll}
o::= & b \mid o_{v} & \\
& o \bullet \bullet o^{\prime} & \text { sequential composition } \\
& o \stackrel{m}{m^{\prime}} o^{\prime} & \text { probabilistic choice } \\
& \forall\left(o \| o^{\prime}\right) & \text { all-to-finish (a.k.a. last-to-finish) } \\
& \exists\left(o \| o^{\prime}\right) & \text { first-to-finish }
\end{array}
$$

We take o $\| o^{\prime}$ to be commutative.

In Section 4, we used these syntax elements as follows:

- $b$ in Section 4.1.

- $o_{v}$ and $o \bullet \bullet \bullet o^{\prime}$ throughout Section 4.

- $o \stackrel{\frac{m}{m^{\prime}}}{o^{\prime}}$ in Section 4.3.

- $\forall\left(o \| o^{\prime}\right)$ in Sections 4.6 and 4.7 .

- $\exists\left(o \| o^{\prime}\right)$ in Section 4.7 .

Definition 2. The evaluation contexts $\mathcal{C}$ of an outcome are defined as follows:

$$
\mathcal{C}::=[]|\mathcal{C} \bullet \rightarrow \bullet| o \bullet \rightarrow \mathcal{C}\left|\mathcal{C} \underset{m^{\prime}}{\stackrel{m}{m}} o\right| o \underset{m}{\stackrel{m}{m^{\prime}}} \mathcal{C}|\forall(\mathcal{C} \| o)| \exists(\mathcal{C} \| o) .
$$

where "[]" is the empty context.

Evaluation contexts are useful in the definition of outcome transitions, which we define next. 
Definition 3. Outcome transitions $\tau_{0}: o \rightarrow o^{\prime}$ are defined by the following rewrite rules:

$$
\begin{array}{llr}
\mathcal{C}[b] \rightarrow \mathcal{C}[o] & o \notin \mathbb{B} & \text { (UNBX) } \\
\mathcal{C}\left[o_{v}\right] \rightarrow \mathcal{C}[o] & o \notin \mathbb{B} & \text { (ELAB) } \\
\mathcal{C}[o] \rightarrow \mathcal{C}\left[o^{\prime} \stackrel{m}{=} o^{\prime \prime}\right] & \text { for some } m, m^{\prime} \in \mathbb{R}^{+}, o^{\prime}, o^{\prime \prime} \in \mathbb{O} & \text { (PROB) } \\
\mathcal{C}[o] \rightarrow \mathcal{C}\left[\forall\left(o^{\prime} \| o^{\prime \prime}\right)\right] & \text { for some } o^{\prime}, o^{\prime \prime} \in \mathbb{O} & (\mathrm{A} 2 \mathrm{~F}) \\
\mathcal{C}[o] \rightarrow \mathcal{C}\left[\exists\left(o^{\prime} \| o^{\prime \prime}\right)\right] & \text { for some } o^{\prime}, o^{\prime \prime} \in \mathbb{O} & \text { (F2F). }
\end{array}
$$

Formally speaking, a refinement step is an instance of an outcome transition. The formal description of the system is refined when one or more refinement steps are taken.

The restriction on (UNBX) is because it makes no sense to replace a black box with another black box. (See the trailing discussion of Section 3.1 on the intention behind black boxes.) The restriction on (ELAB) is because it makes no sense for an outcome variable to be replaced by another outcome variable or a black box.

Considering Definition 3 to be part the syntax is unusual. After all, evaluation contexts are a formalism for the semantics of programming languages. However, for $\triangle Q S D$, it turns out that the rewrites only cause syntactic changes to the outcome expressions (and the corresponding diagrams). Note that a refinement is not a system evolution, but rather, an update in the system description. It is only at analysis time that one tries to understand the meaning of an outcome diagram/expression.

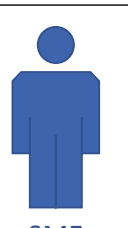

SME

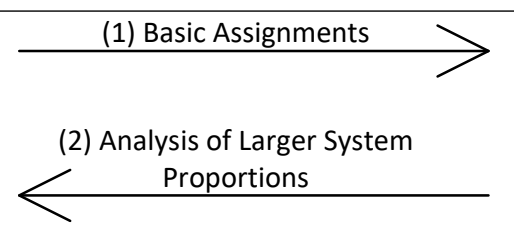

(2) Analysis of Larger System

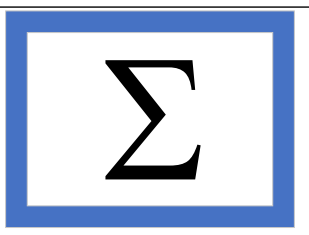

Analysis Formulation

Figure 16. For an analysis, the design engineer provides basic assignments and receives more advanced values for larger parts of the system.

\subsection{Timeliness Analysis}

We are now ready to describe the process of $\Delta Q$ analysis. The idea is that the design engineer provides the basic $\Delta \mathrm{Q}$ analysis to the formulation in Definition 4. Our formulation then enables them to determine the $\Delta \mathrm{Q}$ analysis of the larger parts of their system, or even all of it. This formulation is both compositional and simple. We call the $\Delta Q$ analysis that is provided by the design engineer the basic $(\Delta Q)$ assignment (Definition 4 ). In the basic assignment, the design engineer only maps $\overline{\mathbb{B}}$ expressions. They map those expressions to either CDFs or $\Delta \mathrm{Q}$ variables. In return, they receive more complex $\Delta \mathrm{Q}$ expressions. This is shown in Figure 16. The process is similar for load analysis, except that, there, the values exchanged between the design engineer and the respective formulation refer instead to static amounts of work.

The reason for including the CDFs in the input type of basic assignments is rather obvious. The choice to allow $\Delta Q$ variables here might be less so. The assignment of those $\overline{\mathbb{B}}$ expressions that are mapped to $\Delta Q$ variables are considered to be left by the design engineer for later. As such, the formulation in Definition 4 takes the $\Delta Q$ value of those expressions to be $T$. That is to let the design engineer investigate feasibility even when those particular expressions are disregarded for the moment. 
Fix a set $\Gamma \ni \gamma$ of all CDFs. Fix also a countable set of $\Delta Q$ variables $\Delta_{v} \ni \delta_{v}$. Let $\Delta=\Delta_{v} \cup \Gamma$, where $\Delta \ni \delta$.

Definition 4. Given a basic assignment $\Delta_{\circ}[\cdot]: \overline{\mathbb{B}} \rightarrow \Delta$, define $\Delta Q\left[\cdot \rrbracket_{\Delta_{\circ}}: \mathbb{O} \rightarrow \Gamma\right.$ such that

$$
\begin{aligned}
& \Delta Q \llbracket \beta \rrbracket_{\Delta_{\circ}} \quad= \begin{cases}\top & \text { when } \Delta_{\circ} \llbracket \beta \rrbracket \notin \Gamma \\
\Delta_{\circ} \llbracket \beta \rrbracket & \text { otherwise }\end{cases} \\
& \Delta Q \llbracket o \bullet \longrightarrow o^{\prime} \rrbracket_{\Delta_{\circ}}=\Delta Q \llbracket o \rrbracket_{\Delta_{\circ}} \oplus \Delta Q\left[o^{\prime} \rrbracket_{\Delta_{\circ}}\right. \\
& \Delta Q \llbracket o \stackrel{\frac{m}{m}}{m} o^{\prime} \rrbracket_{\Delta_{\circ}}=\frac{m}{m+m^{\prime}} \Delta Q \llbracket o \rrbracket_{\Delta_{\circ}}+\frac{m^{\prime}}{m+m^{\prime}} \Delta Q \llbracket o^{\prime} \rrbracket_{\Delta_{\circ}} \\
& \Delta Q\left[\forall\left(o \| o^{\prime}\right) \rrbracket_{\Delta_{\circ}}=\max \left(\Delta Q \llbracket o \rrbracket_{\Delta_{\circ}}, \Delta Q \llbracket o^{\prime}\right]_{\Delta_{\circ}}\right) \\
& \Delta Q\left[\exists\left(o \| o^{\prime}\right) \rrbracket_{\Delta_{\circ}}=\min \left(\Delta Q \llbracket o \rrbracket_{\Delta_{\circ}}, \Delta Q \llbracket o^{\prime} \rrbracket_{\Delta_{\circ}}\right)\right. \\
& \text { where } \oplus \text { denotes the convolution of two } \Delta Q s
\end{aligned}
$$

We denote the set of all basic assignments by $\left\{\Delta_{\circ}[\cdot]\right\}$.

We demonstrated the use of this definition in Section 4.2. In programming language theory, Definition 4 is said to give a denotational semantics for $\mathbb{O}$. This is because the formulation works by compositionally denoting the $\mathbb{O}$ syntax into a familiar domain, which is deemed to be simpler $(\Gamma$ here), for example. Definition 4 gives the design engineer the possibility of determining the $\Delta \mathrm{Q}$ behaviour of a snapshot of their system. Armed with that information, the design engineer needs to figure out whether such $\Delta \mathrm{Q}$ behaviour is affordable. In other words, they need to make sure the actual $\Delta \mathrm{Q}$ is within the acceptable bounds. In order to do that, we assume that the design engineer's customer will provide them with a demand CDF: one that defines the acceptable bounds. Definition 5 below is a recipe for comparing the actual behaviour against a demand CDF.

Definition 5. Given a demand CDF $\gamma$ and a partial order $<$ on $\Gamma$, say that a basic assignment $\Delta_{\circ}$ is a witness that an outcome $o$ is a hazard w.r.t. $\gamma$

$$
\Delta_{\circ} \mid=<\operatorname{hazard}_{\gamma}(o)
$$

when

$$
\Delta Q \llbracket o \rrbracket_{\Delta_{\circ}} \nless \gamma .
$$

Likewise, say $\Delta_{\circ}$ is a witness that an outcome o has slack once compared with $\gamma$

$$
\Delta_{\circ} \models<\operatorname{slack}_{\gamma}(o)
$$

when

$$
\Delta Q \llbracket o \rrbracket_{\Delta_{\circ}}<\gamma
$$

The formulation of Definition 5 enables the design engineer to perform the $\Delta Q$ analysis of a single snapshot of their system. In some cases that is enough because it can, for example, reveal the absolute infeasibility of a design. For the majority of cases, however, it is not enough. After all, a snapshot $\Delta \mathrm{Q}$ analysis might not be conclusive, for a variety of reasons. For example, one might not see any indication of a hazard by employing just Definition 5 because more detail is required. That takes us to Definition 8. When a design engineer works out the $\Delta \mathrm{Q}$ analysis of a snapshot, the results might be favourable at the given level of refinement but still inaccurate. In such a case, a design engineer may wish to refine the system and perform the snapshot $\Delta \mathrm{Q}$ again to check whether the refinement confirms the initial $\Delta Q$ analysis. Definition 8 examines that overall confirmation. Definitions 6 and 7 set the stage. 
Definition 6. Let $\Delta_{\circ}$ be a basic assignment. Write

$$
D_{\Gamma}\left(\Delta_{\circ}\right)=\left\{\beta \in \overline{\mathbb{B}} \mid \Delta_{\circ}(\beta) \in \Gamma\right\}
$$

for those $\overline{\mathbb{B}}$ outcomes in the domain of $\Delta_{\circ}$ that $\Delta_{\circ}$ maps to CDFs.

Definition 7. Say $\Delta_{\circ}^{\prime}$ refines $\Delta_{\circ}\left(\right.$ write $\left.\Delta_{\circ} \rightarrow_{\Delta} \Delta_{\circ}^{\prime}\right)$ when

- $D_{\Gamma}\left(\Delta_{\circ}\right) \subseteq D_{\Gamma}\left(\Delta_{\circ}^{\prime}\right)$

- $\forall \beta \in D_{\Gamma}\left(\Delta_{\circ}\right) . \Delta_{\circ}(\beta)=\Delta_{\circ}^{\prime}(\beta)$.

In such a case, call $\Delta_{\circ} \rightarrow_{\Delta} \Delta_{\circ}^{\prime}$ a $\Delta Q$ refinement. When clear, we will replace $\rightarrow_{\Delta}$ by $\rightarrow$.

In words, a basic assignment refines another one when it keeps all the CDFs in place and possibly adds more. We are now ready for Definition 8.

Definition 8. Fix an outcome transition $o \rightarrow o^{\prime}$ and a $\Delta Q$ refinement $\Delta_{\circ} \rightarrow \Delta_{\circ}^{\prime}$. Given a partial order $<$ on $\Gamma$, we say that $\Delta_{\circ} \rightarrow \Delta_{\circ}^{\prime}$ witnesses that $o \rightarrow o^{\prime}$ arms a hazard

$$
\Delta_{\circ} \rightarrow \Delta_{\circ}^{\prime} \models<\operatorname{hazard}\left(o \rightarrow o^{\prime}\right)
$$

when $\Delta Q \llbracket o \rrbracket_{\Delta_{\circ}} \nless \Delta Q\left[o^{\prime} \rrbracket_{\Delta_{\circ}^{\prime}}\right.$. Likewise, say $\Delta_{\circ} \rightarrow \Delta_{\circ}^{\prime}$ witnesses that $o \rightarrow o^{\prime}$ leaves the system slack

$$
\Delta_{\circ} \rightarrow \Delta_{\circ}^{\prime} \models<\operatorname{slack}\left(o \rightarrow o^{\prime}\right)
$$

when $\Delta Q \llbracket o \rrbracket_{\Delta_{\circ}}<\Delta Q \llbracket o^{\prime} \rrbracket_{\Delta_{\circ}^{\prime}}$.

As can be seen from Definitions 5 and 8, all the decisions for the timeliness analysis are made by scrutinising the CDFs (which represent $\Delta Q$ values). This is a consequence of the simple denotational semantics of Definition 4. The fact that the latter formalism is denotational implies that comparisons can be made in the domain of CDFs. Moreover, these comparisons are affordable because the denotational semantics is simple (despite being effective).

\subsection{Load Analysis}

This section describes how the same approach can be used to analyse the load on given resources. Resources can be of different types, in particular we distinguish ephemeral resources that are available at a certain rate, and fixed resources that are available in a fixed number or amount. Examples of ephemeral resources are CPU cycles, network interface capacity, and disk IO operations. Fixed resources include CPU cores, memory capacity and disk capacity. In this paper, we consider only ephemeral resources. The analysis that we want is an answer to the following question: will the resource manage the amount of work assigned to it in the available time frame?

We first need to set up some terminology for specifying the available time frame as well as the amount of work that is assigned to a given resource. Write $t^{\circ}(o)$ for the time an observable from the starting set of an outcome o occurs. Let $t^{\infty}(o)=t^{\circ}(o)+d(o)$, where $d(o)$ denotes the duration limit of $o$. Fix a set of resources $\mathbb{H} \ni \rho$. Note that the amount of work that is assigned to a resource $\rho$ is not scalar. It is, of course, necessary to provide the unit of measurement. For example, when $\rho$ represents CPU resources, a sensible unit of measurement is the number of CPU cycles. When $\rho$ represents network resources, a sensible unit of measurement is the message size. At the current level of formalisation, however, we wish to set ourselves free from thinking about units of measurement. Therefore, given a resource $\rho$, we write $W_{\rho}$ for the set of values of the right unit of measurement for an amount of work that has been assigned to $\rho$.

The design engineer utilises our load analysis in the same way that they utilise our $\Delta \mathrm{Q}$ analysis. That is, they must provide some basic load analysis (Definition 9). Then, exactly as shown in Figure 16, 
they use the formulation in Definition 10 to determine the load analysis for larger parts of their system, or possibly all of it. We now formalise what we mean by a basic load analysis.

Definition 9. For a given $\rho$, a basic "static (amount of) work assignment for $\rho$ " is a function:

$$
\left.\rho \| \frac{W}{} S_{\circ} \llbracket \cdot\right]: \overline{\mathbb{B}} \rightarrow W_{\rho}
$$

Definition 10. Given a basic static work assignment $S_{\circ}$ for $\rho$, the static work assignment (i.e., the amount of work to perform a single outcome per unit of size)

$$
\rho \| W\left[\rrbracket_{S_{\circ}}(.): \mathbb{O} \rightarrow T \rightarrow W_{\rho}\right.
$$

(where $T$ stands for time) is defined as

$$
\begin{aligned}
& \rho \| W \\
& \rho \| W S \llbracket o \bullet \bullet o^{\prime} \rrbracket S_{\circ}(t)=\left\{\begin{array}{l}
\rho \| W \\
\rho \| W \llbracket S \llbracket \rrbracket_{S_{\circ}}(t) \\
\rho \| o^{\prime} \rrbracket_{S_{\circ}}(t)
\end{array}\right.
\end{aligned}
$$

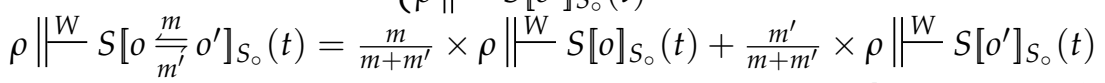

$$
\begin{aligned}
& t \in\left[\min \left(t^{\circ}(o), t^{\circ}\left(o^{\prime}\right)\right), \max \left(t^{\circ}(o), t^{\circ}\left(o^{\prime}\right)\right)\right] \\
& \rho \| W=
\end{aligned}
$$

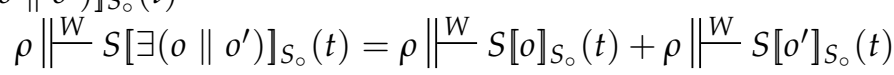

$$
\begin{aligned}
& t \in\left[\min \left(t^{\circ}(o), t^{\circ}\left(o^{\prime}\right)\right), \max \left(t^{\circ}(o), t^{\circ}\left(o^{\prime}\right)\right)\right]
\end{aligned}
$$

Whether or not a given resource $\rho$ is overloaded when performing an outcome $o$ is determined by whether $\rho$ can bear the offered load in the required duration, $d(o)$. The smaller that $d(o)$ is, the faster (i.e., the more intensely) $o$ must be performed. However, that can only be done up to a certain threshold that is determined by the system's configuration. In other words, whether the intensity brought to $\rho$ passes a given threshold is what determines whether $\rho$ is overloaded. As with $W_{\rho}$, at our current level of abstraction, we wish to disregard the units of measurement for intensity. That is, we write $I_{\rho}$ for the set of values of the right unit of measurement for the intensity of the load that is imposed on $\rho$. We single out $\theta_{I}(\rho) \in I_{\rho}$ for the threshold of intensity $\rho$ can bear. When it is clear, we write $\theta_{I}$ for $\theta_{I}(\rho)$.

Definition 11. For a fixed $\rho$, given a threshold of intensity $\theta_{I}(\rho)$ and a basic static work assignment $S_{\circ}$ for $\rho$, the static slack of an outcome in $\rho$-consumption:

$$
S_{\circ} \models_{\rho} \operatorname{slack}_{\theta_{I}}(.): \mathbb{O} \rightarrow T \rightarrow I_{\rho}
$$

is defined as

$$
S_{\circ} \models \rho \operatorname{slack}_{\theta_{I}}(o)=\theta_{I}-\frac{\rho \| W}{d(o)} S \llbracket o \rrbracket_{S_{\circ}} .
$$

Define the static hazard of an outcome in $\rho$-consumption:

$$
S_{\circ}=\rho \operatorname{hazard}_{\theta_{I}}(o)=-S_{\circ}=\rho \operatorname{slack}_{\theta_{I}}(o) \text {. }
$$

Our emphasis on considering the analyses of Definitions 9-11 "static" is intentional. Firstly, they all assume that a base outcome's work is spread uniformly over its duration limit. That is obviously not always correct. The work assignment typically varies over the duration limit. However, if to every base outcome $\beta$, the design engineer chooses to assign the highest amount of work that $\beta$ needs to do 
during its duration limit, the analyses given in Definition 11 would lead to a safe upper bound, that is useful as a first estimate. Secondly, Definitions 9-11 assume that an outcome's amount of work is always the same throughout its execution. Again, that is not realistic. Various reasons might cause the amount of work assigned to a base outcome to change over time. Examples are congestion, nonlinear correlations between outcomes, and cascading effects. This suggests more advanced load analyses that are "dynamic" rather than the "static" ones we have described here. We leave the development of such analyses to future work.

\section{Related Work}

Several theoretical or practical approaches have previously been proposed that address parts of the problem that has been identified above, but none of these addresses the whole problem in a comprehensive way.

\subsection{Alternative Theoretical Approaches}

\subsubsection{Queuing theory}

Steady-state performance has been widely studied as an aid to analysis, for example in queuing theory. Such approaches tend to take a resource-centric view of the system components, focusing on their individual utilisation/idleness. Where job/customer performance is considered, such as in mean-value analysis [19] or Jackson/BCMP networks [20], it is also in the context of steady-state averages. However, these traditional approaches cannot deliver metrics such as the time distribution of the system's response to an individual stimulus or even the probability that such a response will occur within a given time bound. These metrics are key for any time-critical and/or customer-experience-centric service.

\subsubsection{Extending existing modelling approaches}

With the exception of hard real-time systems, it is rare to see performance treated as a "first-class citizen" in a system design process. At best, performance is considered as a property that will emerge during the system development life-cycle, and thus something that can only be retrospectively validated. In contrast with $\triangle \mathrm{QSD}$, performance is thus unverifiable when using such an approach.

A common approach has been to extend existing approaches to modelling distributed systems, such as Petri nets or process calculi, with additional features with the goal of integrating performance modelling into existing design processes. Examples include stochastic Petri nets [21], timed and probabilistic process calculi [22,23] and performance evaluation process algebra (PEPA [24]). These systems consider passage-time [25], the time taken for the system to follow a particular path to a state, that path being characteristic of an outcome of interest [26-29]. As described above, these are all retrospective validation tools, requiring fully specified systems, that will give probabilistic measures of outcomes under steady-state assumptions. These systems are susceptible to state space explosion as a model grows in complexity, and this therefore limits their usage to less complex systems. Furthermore, like queuing models, they do not model failure, nor do they model typical real-world responses to failure such as timeouts and retries.

\subsubsection{Real-time systems and Worst-case Execution Time}

In real-time systems actions must be completed by strict deadlines. Missed deadlines can be catastrophic (hard real-time systems) or lead to significant delay and loss caused by roll-backs or recovery (soft real-time systems). Performance analysis has focused on giving guarantees that deadlines can be met by studying worst case execution time [30]. These approaches generally aim to analyse the behaviour of specific implementations, providing information about specific interactions. This approach is thus complementary to design-time approaches such as $\triangle \mathrm{QSD}$. 


\subsection{Distributed System Design}

Designing large distributed systems is costly and error-prone. This might seem paradoxical given the proliferation of modern Internet-based companies whose core business is based on large distributed systems, such as Google, Facebook, Amazon, Twitter, Netflix and many others. Given the existence of these successful companies, it might seem that building large distributed systems is a solved problem. It is not. Successful companies have built their systems over many years, using vast amounts of effort and ingenuity to find usable solutions to difficult problems.

\subsubsection{Iterative design}

There does not exist a standard approach for designing large distributed systems that allows prediction of high-load performance early on during the design process. We explain the problem by giving an overview of the current design approach for distributed systems. The approach is iterative. It starts with a specification of the system's desired performance and scale. The system architecture is then designed by determining the system components according to the system's scale and estimating the performance they must have to give the required overall performance. The next step is performance validation, to verify that the design satisfies the performance requirements.

\subsubsection{Testing and simulation}

Performance validation is performed either as part of unit, subsystem and/or system testing, or via discrete-event simulation. Testing the performance of a component or subsystem is inconclusive without a reliable means to relate it to the resulting system performance, and testing of the whole system only reveals issues very late in the system development life-cycle. It is good practice to perform integration testing at this late stage. However, this is a poor and expensive substitute for performance analysis throughout the development process. Simulation can be performed earlier in the development process, and may be less costly than testing, but it is limited in its ability to expose rare cases, and hence cannot test tight bounds on the performance.

In the final analysis, obtaining reliable performance numbers at high load requires actually building a large part of the final system and subjecting it to a realistic load. If the system does not satisfy the requirements, then it is back to the drawing board. The system architecture is redesigned to remove observed and predicted bottlenecks and rebuilt.

\subsubsection{Development risks}

Several iterations of the design may be necessary until the system behaves satisfactorily. It often happens that the system only behaves satisfactorily at a fraction of the required load, but because of market constraints, this is considered acceptable and the system is deployed. In parallel to the deployment, the design engineers continue to work on a system that will accept the larger load, under the assumption that the deployment will be successful so that the load will increase.

This methodology is workable, but it is highly risky due to its high cost and development time. To have a good chance of success, it requires experienced developers. The development budget may be exhausted before achieving a satisfactory system; it may even be determined that the requirements are impossible to satisfy (infeasibility). If this is discovered early on, then the company may be able to retarget itself to become viable. Otherwise, the company simply folds.

\subsubsection{Role of the $\triangle \mathrm{QSD}$ paradigm in distributed system design}

The $\triangle$ QSD paradigm is designed specifically to reduce cost and development time. The system is designed as a sequence of increasingly refined outcome diagrams. At each stage, performance is computed using the $\Delta \mathrm{Q}$ parameters. If the system is infeasible, this is detected early on and it is immediately possible to change the design. If the design has sufficient slack, then the design process continues. The $\triangle \mathrm{QSD}$ paradigm is effective insofar as the $\Delta \mathrm{Q}$ computations provide realistic 
results. This depends on: i) having correct $\Delta \mathrm{Q}$ distributions for the basic components; and ii) correctly specifying causality and resource constraints. Experience with $\triangle \mathrm{QSD}$ in past industrial designs gives us confidence in the validity of the results. The additional rigour that is provided by the $\triangle \mathrm{QSD}$ formalism that has been introduced in this paper gives us confidence that the paradigm is being applied correctly and allows the paradigm to be integrated into new design tools.

\subsection{Programming Languages and Software Engineering}

\subsubsection{Programming Paradigms}

Programming paradigms each focus on their particular discipline for bringing more opportunities for code reuse. The most familiar examples are perhaps Object-Oriented Programming, Functional Programming, and Genericity by Type, which promote code-reuse between a base class and derived ones, by refactoring into functions, and type parameterisation. Gibbons [31] has an excellent survey on different flavours of Generic Programming with the different opportunities for code reuse that each provides. Some programming paradigms have widely-accepted formalisms and some do not. Regardless of the underlying programming paradigm, $\triangle \mathrm{QSD}$ is a paradigm for systems development rather than simply for programming, and comes with its own formalism.

\subsubsection{Software Development Paradigms}

Three paradigms focus on the process of software development and are, hence, closer to $\triangle \mathrm{QSD}$ :

Design-by-Contract. [32] Similarly to $\Delta \mathrm{QSD}$, in this paradigm, the programmer begins by coding by describing the pre-conditions and the post-condition. Over the years, the concept of refining initial designs from specification to code has gained increasing weight [33]. Unlike $\Delta$ QSD, the focus, however, is on functional correctness rather than performance.

Software Product Lines. [34] This paradigm targets families of software systems that are closely related and that clearly share a standalone base. The aim is to reuse the development effort of and the code for the base across all the different variations in the family. The similarity with $\triangle \mathrm{QSD}$ is that this approach also allows variation in the implementation so long as the required quality constraints are met. In other words, variations can share a given expected outcome and its quality bounds.

Component-Based Software Engineering. [35] Components, in this paradigm, are identified by their so-called 'requires' and 'provides' interfaces. That is, so long as two components have the same 'requires' and 'provides' interfaces, they are deemed equivalent in this paradigm, and, can be used interchangeably. In $\triangle \mathrm{QSD}$, subsystems can also have quality contracts that involve quantitative 'demand' and 'supply' specifications. Such contracts impose quality restrictions (say, timeliness or pattern of consumption) on the respective outcomes of those subsystems. We have not shown examples of quality contracts in this paper, however, because their formalisation is not yet complete.

\subsubsection{Algebraic Specification and Refinement}

Algebraic specification languages such as CLEAR [36], Extended ML [37], and CASL [38] work on the basis of specifying requirements using algebraic signatures and equations that are then refined progressively until one makes it to the level of actual code. Refinement in such languages is managed using various media, for example by module systems with rigorously defined formal semantics. Whilst the focus of such languages is almost exclusively on functional correctness, studying possibilities for enhancing algebraic specifications so that they also accommodate the quality of outcomes would be an interesting avenue for future work. 


\subsubsection{Amortised Analysis}

Amortised resource analysis is an approach for promoting resource analysis as a first-class citizen of programming languages specification. Various operational semantics, type systems, and category theoretical approaches have been employed. See [39], [40], and [41], for example, where memory consumption for functional languages such as HASKELL and ML are automatically calculated for programs written in those languages. $\triangle \mathrm{QSD}$ advises on specification at the much higher level of outcomes and outcome diagrams, leaving the actual implementation and its host language completely loose. As a result, $\triangle Q S D$ is much more flexible and also fruitful in swift performance workout throughout the system development life-cycle.

\section{Conclusions}

This paper has presented the $\triangle \mathrm{QSD}$ systems development process, that is steered by performance predictability concerns, and that is supported by a rigorous formalism (Section 5). Our formalisation of $\triangle$ QSD is a part of a wider initiative both within PNSol and IOHK [10]. $\triangle$ QSD has been successfully used in a wide range of industries, including telecommunications, avionics, space and defence, and cryptocurrency. It complements other approaches that are focused primarily on functional concerns, such as functional programming or model checking.

$\triangle$ QSD is based on taking the observable outcomes of a system as the central point of focus (Section 3.1), capturing the causal dependencies between outcomes in the form of outcome diagrams (Section 3.2). The formalism also describes the process of refining outcome diagrams (Definition 7), as part of a system design process. The formal specification of a system serves as a basis for different analyses such as timeliness (Section 5.3) and behaviour under load (Section 5.4). Although we have illustrated the $\triangle$ QSD paradigm in the context of design refinement, the aim is that these aspects should permeate throughout the complete System Development Life-Cycle. Our formalism builds on the simple concept of quality attenuation $(\Delta Q$, Section 3.3) that captures the notion of performance hazard. This helps early detection of infeasibility, so preventing the waste of resources (financial, people, time and systems).

\subsection{Future work}

Future work will include the development of new analyses for non-ephemeral resources and for dynamic loads, and an extension to non-linear systems in which the load and timeliness are coupled. In parallel, we plan to use our formalism as an intermediate step to better teaching and dissemination of $\Delta$ QSD. We will build tools that will enable us to understand how to track the key observables/outcomes from the the design into the implementation so that they can support ongoing system design and development throughout the system development life-cycle. The wider $\Delta \mathrm{Q}$ framework is also under active development within the international Broadband Forum [42] as a means of characterising quality attenuation associated with networks.

1. Narcisse, E. What Went Wrong With OnLive? Technical report, Kotaku, 2012.

2. Wolverton, T. Exclusive: OnLive assets were sold off for just \$4.8 million. Technical report, 2012.

3. The Verge. OnLive lost: how the paradise of streaming games was undone by one man's ego. Technical report, 2012.

4. $\quad$ Pressman, R.; Maxim, D.B.R. Software Engineering: A Practitioner's Approach; McGraw-Hill, 2014.

5. Krasner, H. The Cost of Poor Software Quality in the US: A 2020 Report. Technical report, CISQ Consortium for Information \& Software Quality, 2020.

6. Perry, D.E.; Wolf, A.L. Foundations for the study of software architecture. ACM SIGSOFT Software Engineering Notes 1992, 17, 40-52.

7. Alford, M.W. A requirements engineering methodology for real-time processing requirements. IEEE Transactions on Software Engineering 1977, 1, 60-69. 
8. Solutions, P.N. Assessment of traffic management detection methods and tools. Technical Report MC-316, Ofcom, 2015.

9. Thompson, P.; Davies, N. Towards a RINA-Based Architecture for Performance Management of Large-Scale Distributed Systems. Computers 2020, 2.

10. Kant, P.; Hammond, K.; Coutts, D.; Chapman, J.; Clarke, N.; Corduan, J.; Davies, N.; Díaz, J.; Güdemann, M.; Jeltsch, W.; Szamotulski, M.; Vinogradova, P. Flexible Formality Practical Experience with Agile Formal Methods. Trends in Functional Programming; Byrski, A.; Hughes, J., Eds.; Springer International Publishing: Cham, 2020; pp. 94-120.

11. Drescher, D. Blockchain Basics: A Non-Technical Introduction in 25 Steps; Apress: Frankfurt am Main, Germany, 2017.

12. David, B.; Gaži, P.; Kiayias, A.; Russell, A. Ouroboros Praos: An Adaptively-Secure, Semi-synchronous Proof-of-Stake Blockchain. Advances in Cryptology - EUROCRYPT 2018; Nielsen, J.B.; Rijmen, V., Eds.; Springer International Publishing: Cham, 2018; pp. 66-98.

13. Coutts, D.; Davies, N.; Szamotulski, M.; Thompson, P. Introduction to the design of the Data Diffusion and Networking for Cardano Shelley. Technical report, IOHK, 2020.

14. Watts, D.J., Small Worlds: the Dynamics of Networks between Order and Randomness; Princeton University Press, 2003; p. 280.

15. Leon Gaixas, S.; Perello, J.; Careglio, D.; Gras, E.; Tarzan, M.; Davies, N.; Thompson, P. Assuring QoS Guarantees for Heterogeneous Services in RINA Networks with $\triangle \mathrm{Q}$. IEEE International Conference on Cloud Computing Technology and Science (CloudCom). IEEE, 2016, pp. 584-589.

16. Trivedi, K.S. Probability and statistics with reliability, queuing, and computer science applications, 2 ed.; Wiley: New York, NY, USA, 2002.

17. Thompson, P.; Davies, N. Towards a RINA-Based Architecture for Performance Management of Large-Scale Distributed Systems. Computers 2020, 2.

18. Voulgaris, S. Private Communication.

19. Reiser, M.; Lavenberg, S.S. Mean-value analysis of closed multichain queuing networks. Journal of the ACM 1980, 27, 313-322.

20. Jackson, J.R. Jobshop-like queueing systems. Management science 1963, 10, 131-142.

21. Molloy, M.K. Performance analysis using stochastic petri nets. IEEE Transactions on Computers 1982, 31, 913-917.

22. Moller, F.; Tofts, C. A Temporal Calculus for Communicating Systems. CONCUR '90; Baeten, J.C.M.; Klop, J.W., Eds. Springer-Verlag, 1989, Vol. 458, LNCS, pp. 401-415.

23. Jou, C.C.; Smolka, S.A. Equivalences, Congruences and Complete Axiomatizations of Probabilistic Processes. CONCUR '90; Baeten, J.C.M.; Klop, J.W., Eds. Springer-Verlag, 1989, Vol. 458, LNCS, pp. 367-383.

24. Hillston, J. A Compositional Approach to Performance Modelling; Cambridge University Press, 1996.

25. Bradley, J.T.; Dingle, N.J.; Gilmore, S.T.; Knottenbelt, W.J. Derivation of passage-time densities in PEPA models using IPC: The Imperial PEPA Compiler. 11th IEEE/ ACM International Symposium on Modeling, Analysis and Simulation of Computer Telecommunications Systems, 2003, pp. 344-351.

26. Daduna, H. Burke's Theorem on Passage Times in Gordon-Newell Networks. Advances in Applied Probability 1984, 16, 867-886.

27. Guang-Hui, H.; Xue-Ming, Y. First passage times and their algorithms for markov processes. Communications in Statistics. Stochastic Models 1995, 11, 195-210, [https://doi.org/10.1080/15326349508807338]. doi:10.1080/15326349508807338.

28. Bradley, J.; Davies, N. Performance Modelling and Synchronisation. Workingpaper, University of Bristol, United Kingdom, 1998. Other: CSTR-98-009, Superseded by CSTR-99-002.

29. Chatrabgoun, O.; Daneshkhah, A.; Parham, G. On the functional central limit theorem for first passage time of nonlinear semi-Markov reward processes. Communications in Statistics - Theory and Methods 2020, 49, 4737-4750, [https:/ / doi.org/10.1080/03610926.2019.1606917]. doi:10.1080/03610926.2019.1606917.

30. Wilhelm, R.; Engblom, J.; Ermedahl, A.; Holsti, N.; Thesing, S.; Whalley, D.; Bernat, G.; Ferdinand, C.; Heckmann, R.; Mitra, T.; Mueller, F.; Puaut, I.; Puschner, P.; Staschulat, J.; Stenström, P. The Worst-Case Execution-Time Problem-Overview of Methods and Survey of Tools. ACM Trans. Embed. Comput. Syst. 2008, 7. doi:10.1145/1347375.1347389. 
31. Gibbons, J., Ed. Generic and Indexed Programming - International Spring School, SSGIP 2010, Oxford, UK, March 22-26, 2010, Revised Lectures, Vol. 7470, Lecture Notes in Computer Science. Springer, 2012. doi:10.1007/978-3-642-32202-0.

32. Meyer, B. Applying "Design by Contract". Computer 1992, 25, 40-51. doi:10.1109/2.161279.

33. Weigand, H.; Dignum, V.; Meyer, J.J.C.; Dignum, F. Specification by Refinement and Agreement: Designing Agent Interaction Using Landmarks and Contracts. Engineering Societies in the Agents World III, Third International Workshop, ESAW 2002, Madrid, Spain, September 16-17, 2002, Revised Papers; Petta, P.; Tolksdorf, R.; Zambonelli, F., Eds. Springer, 2002, Vol. 2577, Lecture Notes in Computer Science, pp. 257-269. doi:10.1007/3-540-39173-8\_19.

34. Apel, S.; Batory, D.B.; Kästner, C.; Saake, G. Feature-Oriented Software Product Lines - Concepts and Implementation; Springer, 2013. doi:10.1007/978-3-642-37521-7.

35. Pree, W. Component-Based Software Development - A New Paradigm in Software Engineering? Softw. Concepts Tools 1997, 18, 169-174.

36. Baumeister, H. Relating Abstract Datatypes and Z-Schemata. Recent Trends in Algebraic Development Techniques, 14th International Workshop, WADT'99, Château de Bonas, France, 1999, Selected Papers; Bert, D.; Choppy, C.; Mosses, P.D., Eds., 2000, LNCS Vol. 1827, pp. 366-382.

37. Kahrs, S.; Sannella, D.; Tarlecki, A. The Definition of Extended ML: A Gentle Introduction. Theor. Comput. Sci. 1997, 173, 445-484. doi:10.1016/S0304-3975(96)00163-6.

38. Astesiano, E.; Bidoit, M.; Kirchner, H.; Krieg-Brückner, B.; Mosses, P.D.; Sannella, D.; Tarlecki, A. CASL: The Common Algebraic Specification Language 2002. 286, 153-196.

39. Simões, H.R.; Vasconcelos, P.B.; Florido, M.; Jost, S.; Hammond, K. Automatic amortised analysis of dynamic memory allocation for lazy functional programs. ACM SIGPLAN International Conference on Functional Programming, ICFP'12, Copenhagen, Denmark, September 9-15, 2012; Thiemann, P.; Findler, R.B., Eds. ACM, 2012, pp. 165-176. doi:10.1145/2364527.2364575.

40. Jost, S.; Vasconcelos, P.B.; Florido, M.; Hammond, K. Type-Based Cost Analysis for Lazy Functional Languages. J. Autom. Reason. 2017, 59, 87-120. doi:10.1007/s10817-016-9398-9.

41. Rajani, V.; Gaboardi, M.; Garg, D.; Hoffmann, J. A unifying type-theory for higher-order (amortized) cost analysis. Proc. ACM Program. Lang. 2021, 5, 1-28. doi:10.1145/3434308.

42. Thompson, P.; Hernadaz, R. Quality Attenuation Measurement Architecture and Requirements. Technical Report TR-452.1, Broadband Forum, 2020. 